\title{
Intelligent Wind Retrieval from Chinese Gaofen-3 SAR Imagery in Quad Polarization
}

\author{
WeIZENG ShaO AND SHUAi ZHU \\ Marine Science and Technology College, Zhejiang Ocean University, Zhoushan, China \\ XiAOPEng Zhang, Shuiping GoU, AND ChangZhe JiAO \\ School of Artificial Intelligence, Xidian University, Xi'an, China \\ XINZHE YUAN \\ Key Laboratory of Space Ocean Remote Sensing and Application, National Satellite Ocean \\ Application Service, Beijing, China \\ LIANGBO ZHAO \\ Beijing Institute of Spacecraft System Engineering, Beijing, China
}

(Manuscript received 29 March 2019, in final form 13 July 2019)

\begin{abstract}
This study proposes the use of the artificial neural network for wind retrieval with Chinese Gaofen-3 (GF-3) synthetic aperture radar (SAR) data. More than 10000 images acquired in wave mode and quadpolarization strip map were collected over global seas throughout the 2 -yr mission. The $G F-3$ operated in a quad-polarization channel-vertical-vertical (VV), vertical-horizontal (VH), horizontal-horizontal $(\mathrm{HH})$, and horizontal-vertical (HV). These images were collocated with winds from the European Centre for Medium-Range Weather Forecasts at a $0.125^{\circ}$ grid. The newly released wind retrieval algorithm for copolarization (VV and $\mathrm{HH}$ ) SAR included CMOD7 and C-SARMOD2. We developed an algorithm based on an artificial neural network method using the SAR-measured normalized radar cross section at quad-polarization channels, herein named QPWIND_GF. Simulations using the QPWIND_GF showed that the correlation coefficient of wind speed was 0.94 . We then validated the retrieval wind speeds against the measurements at a $0.25^{\circ}$ grid from the Advanced Scatterometer. A comparison showed that the root-mean-square error (RMSE) of wind speed was $0.74 \mathrm{~m} \mathrm{~s}^{-1}$, which was better than the wind speed obtained using state-of-the-art methods-including, for example, CMOD7 (RMSE $0.88 \mathrm{~m} \mathrm{~s}^{-1}$ ) and C-SARMOD2 (RMSE $1.98 \mathrm{~m} \mathrm{~s}^{-1}$ ). The finding indicated that the accuracy of wind retrieval from $G F-3$ SAR images was significantly improved. Our work demonstrates the advanced feasibility of an artificial neural network method for SAR marine applications.
\end{abstract}

\section{Introduction}

Artificial intelligence is a research focus in remote sensing, which is used in many projects around the world. Ocean remote sensing is one of the most important of these applications. A ocean remote sensing system produces a large amount of data, which can profoundly affect the development of ocean applications that use processing tools, including artificial intelligence methods.

Corresponding authors: Weizeng Shao, shaoweizeng@zjou.edu. cn; Shuiping Gou, shpgou@mail.xidian.edu.cn
Recently, synthetic aperture radar (SAR) remote sensing has shown the ability to retrieve sea surface wind fields at a fine spatial resolution and with wide spatial coverage. The China Academy of Space Technology (CAST) launched the C-band Chinese civilian Gaofen-3 (GF-3) SAR satellite in August 2016, which offers 12 alternative imaging modes with a pixel resolution of up to $1 \mathrm{~m}$ (Shao et al. 2018). Among the various modes, wave mode (WV) and quad-polarization strip map (QPS) operate by collecting quad-polarization images, including vertical-vertical (VV), horizontal-horizontal $(\mathrm{HH})$, vertical-horizontal (VH), and horizontal-vertical (HV) 


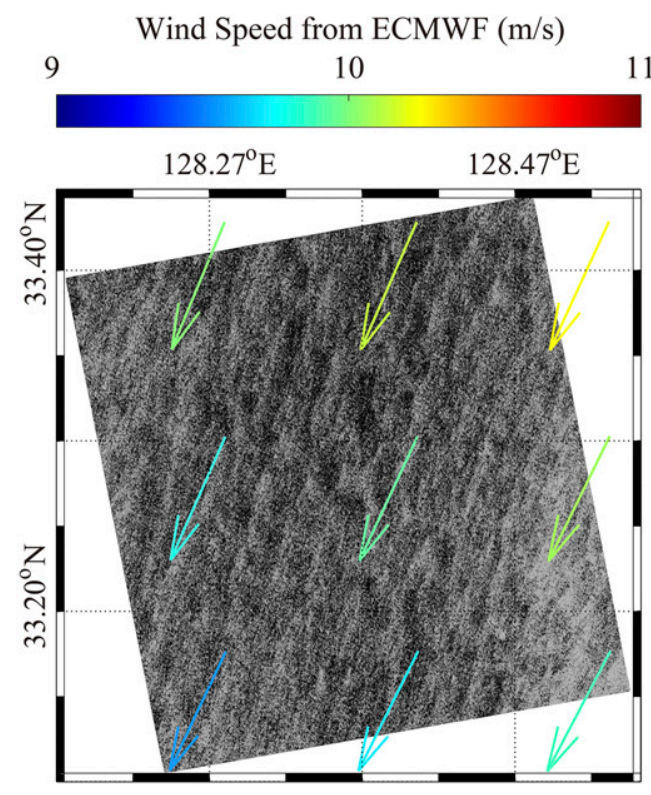

(a)

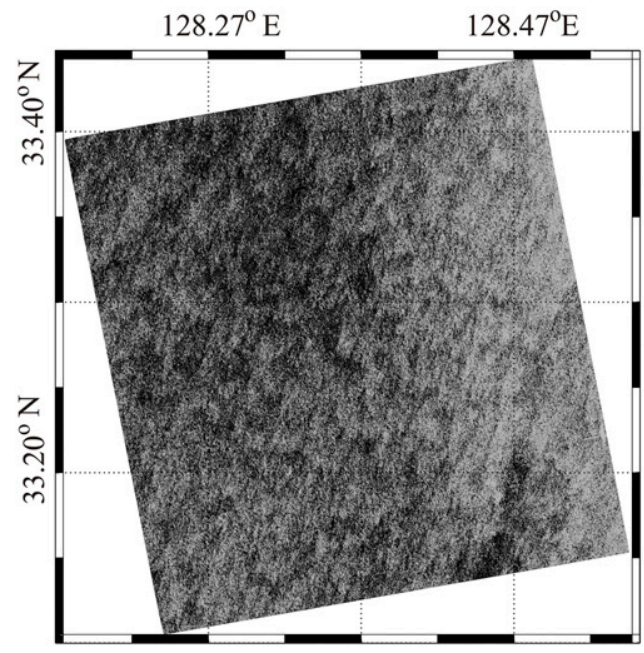

(c)

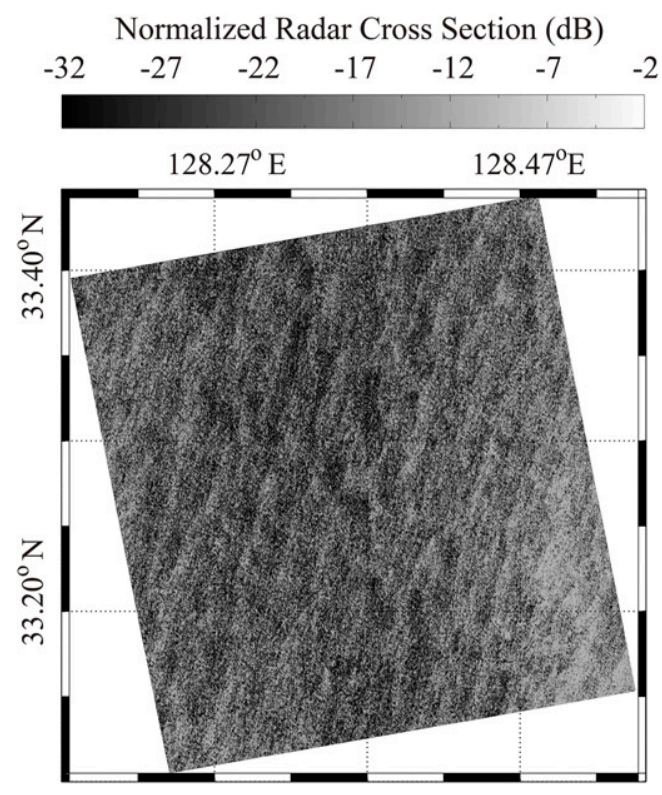

(b)

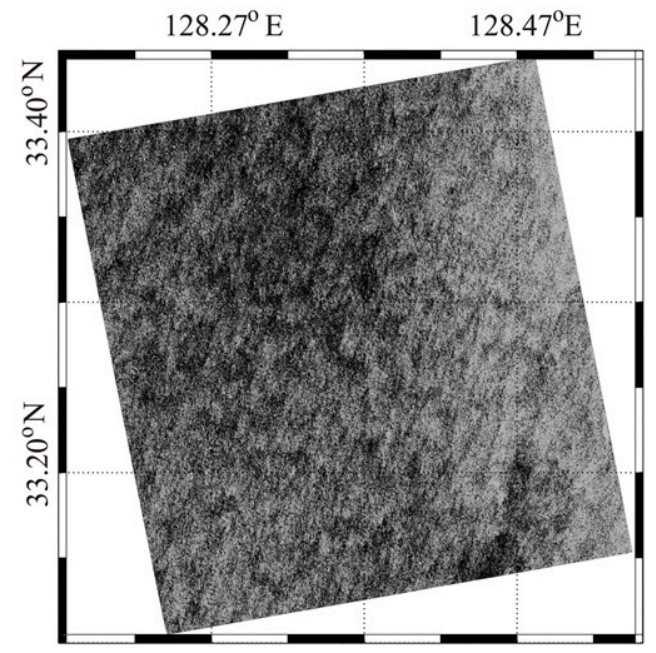

(d)

FIG. 1. The GF-3 SAR image with homogenous wind streaks acquired in QPS mode at 0926 UTC 31 Mar 2017: (a) VV GF-3 image for which colored arrows show the $0.125^{\circ}$ gridded ECMWF reanalysis wind vectors at 0600 UTC, (b) HH GF-3 image, (c) HV GF-3 image, and (d) VH GF-3 image.

images. In particular, the data acquired in wave mode are used frequently to determine the duration of an onorbit calibration for GF-3 SAR (Zhu et al. 2018).

Wind retrieval has been well studied using copolarization (VV and HH) SAR images (Chapron et al. 2001; Lin et al. 2008). Typically, the geophysical model function (GMF) is the scatterometry-based approach commonly used to deal with copolarization SAR images. GMR was originally developed using measurements from a scatterometer and auxiliary wind, for example, European Centre for Medium-Range Weather Forecasts
(ECMWF) reanalysis data. It was found that the GMF also works for SAR wind retrieval. C-band SAR GMFs are denoted as CMOD, and they include CMOD4 (Stoffelen and Anderson 1997), CMOD-IFR developed at the Institut Français de Recherche pour l'Exploitation de la Mer (IFREMER) (Quilfen et al. 1998), CMOD5 (Hersbach et al. 2007; Hersbach 2010), CMOD7 (Stoffelen et al. 2017), C-SARMOD (Mouche and Chapron 2015), and C-SARMOD2 (Lu et al. 2018). An SARbased method to estimate wind speed has also been developed in the literature (Benassai et al. 2018). 


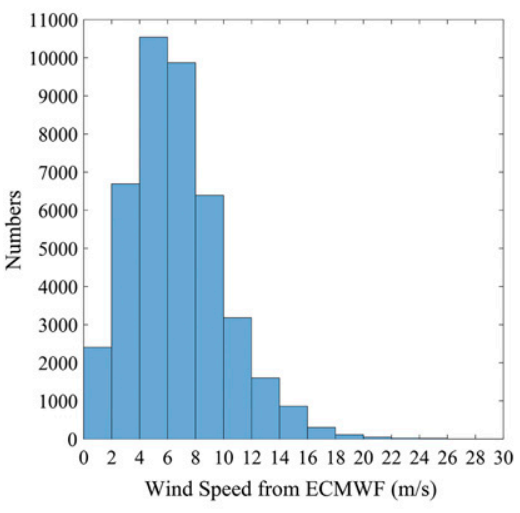

(a)

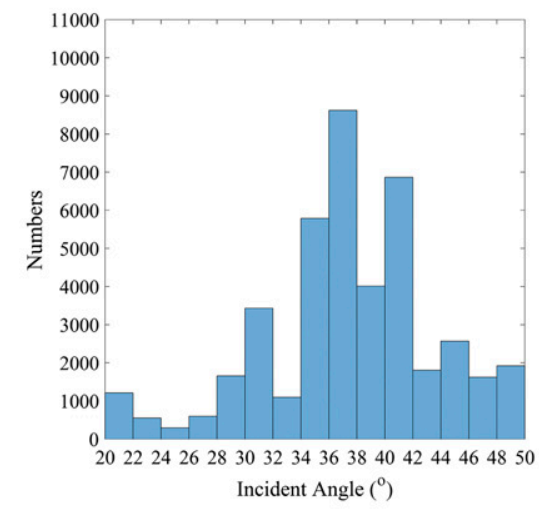

(b)

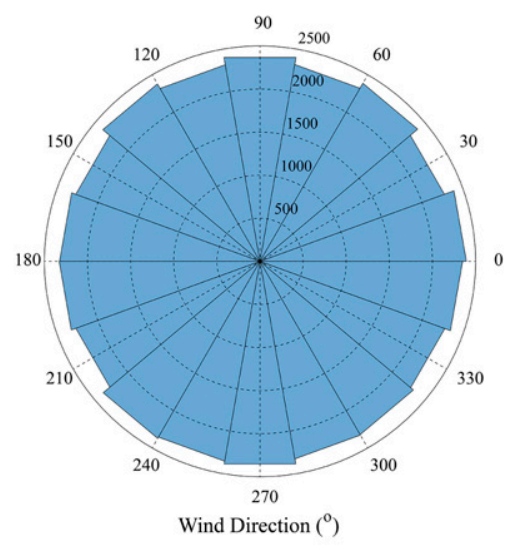

(c)

FIG. 2. Histograms of the (a) ECMWF wind speed and (b) SAR incidence angle, along with (c) a rose diagram of the ECMWF wind direction.

The idea behind this algorithm is that wind speed is related to azimuthal cutoff wavelength (Grieco et al. 2016). It has also been proved that this algorithm shows a good performance under strong wind conditions (Corcione et al. 2019, 2018). All of these GMF algorithms follow the canonical relationship between wind vector and SARmeasured normalized radar cross section (NRCS) at a VV-polarization channel. In addition, the specific functional form of the GMF changes. A polarization ratio (PR) model is commonly used together with the GMF for wind retrieval from an $\mathrm{HH}$-polarization SAR image, in which the HH-polarization NRCS can be directly converted into VV-polarization NRCS (Vachon and Dobson 2000; Horstmann et al. 2000; Mouche et al. 2005; Zhang et al. 2011; Shao et al. 2016). A single NRCS may correspond to a number of wind speed and direction pairs with ambiguity. The fundamental technique to infer wind direction is the detection of linear features on SAR imagery, which is caused by a circulating pattern of local wind rows. These periodic lines are aligned with wind direction that has an inherent $180^{\circ}$ ambiguity (Alpers and Brümmer 1994; Zhou et al. 2017), and external information is employed to remove that ambiguity. The limitation of using linear features for wind direction retrieval, however, is that such features usually are contaminated by other atmospheric and marine phenomena on the same spatial scales (e.g., atmospheric gravity wave, upwelling and eddy).

Recently, several studies have proposed empirical wind retrieval algorithms using cross-polarization ( $\mathrm{VH}$ and HV) SAR NRCS (Hwang et al. 2015; Wang et al. 2018), because the cross-polarization NRCS has a linear growth relationship with wind speed and is less sensitive to wind direction (Vachon and Wolfe 2011;
Ren et al. 2017). The SAR backscattering signal at crosspolarization channel does not easily encounter a saturation problem at strong winds (probably greater than $25 \mathrm{~m} \mathrm{~s}^{-1}$ ) (Hwang et al. 2010; Voronovich and Zavorotny 2014; Zhang and Perrie 2012; Shao et al. 2017). Under these circumstances, the cross-polarization retrieval algorithm demonstrates better performance in typhoon and hurricane conditions (Zhang et al. 2017; Shao et al. 2018).

To date, the accuracy of retrieval wind using existing algorithms for various SAR at copolarization (Yang et al. 2011; Shao et al. 2014; Monaldo et al. 2016) and crosspolarization (Wang et al. 2018; Vachon and Wolfe 2011; Huang et al. 2017) channels has been well studied, indicating a $2 \mathrm{~m} \mathrm{~s}^{-1}$ error of wind speed. Hence, a quad-polarization backscattering signal should include additional sea surface information, which is expected to improve the wind retrieval performance (Fois et al. 2015). Many of the most popular data processing techniques include data mining, machine learning, artificial intelligence, and data fusion (Bello-Orgaz et al. 2016). Many people use artificial intelligence in various fields, and have achieved great success. Within this context, in this study, we used both co- and cross-polarization NRCS to retrieve wind from $G F-3$ SAR based on an artificial neural network from intelligence methods to improve SAR-derived wind accuracy.

The rest of this study is organized as follows: A brief description of the dataset is provided in section 2 (e.g., GF-3 SAR images and auxiliary wind data). The selected four neural network methods and development of intelligent wind retrieval algorithm are introduced in section 3. The experiments and validations of the proposed intelligent algorithm and existing two conventional 


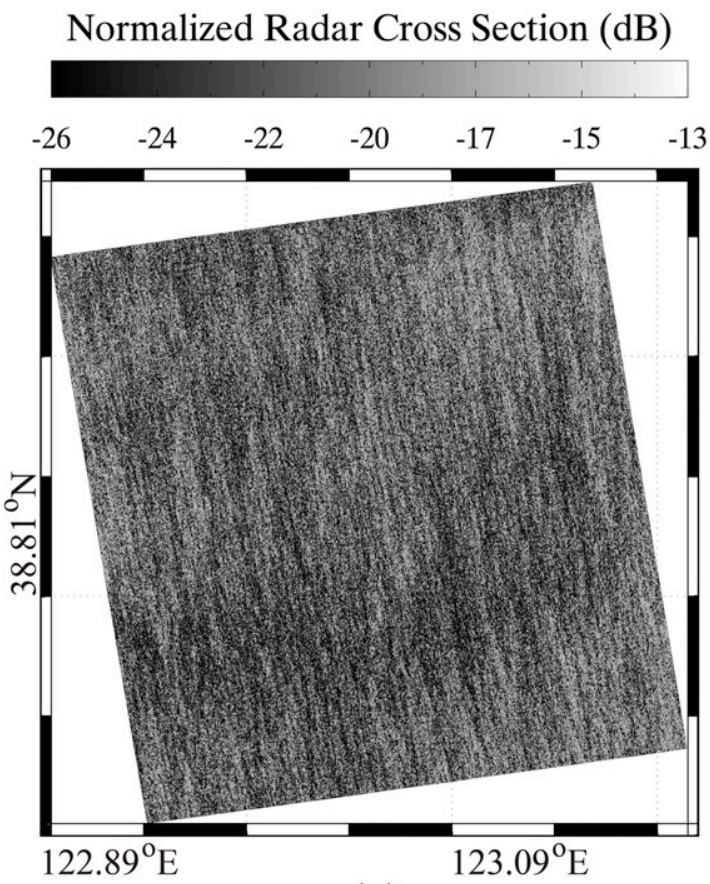

(a)

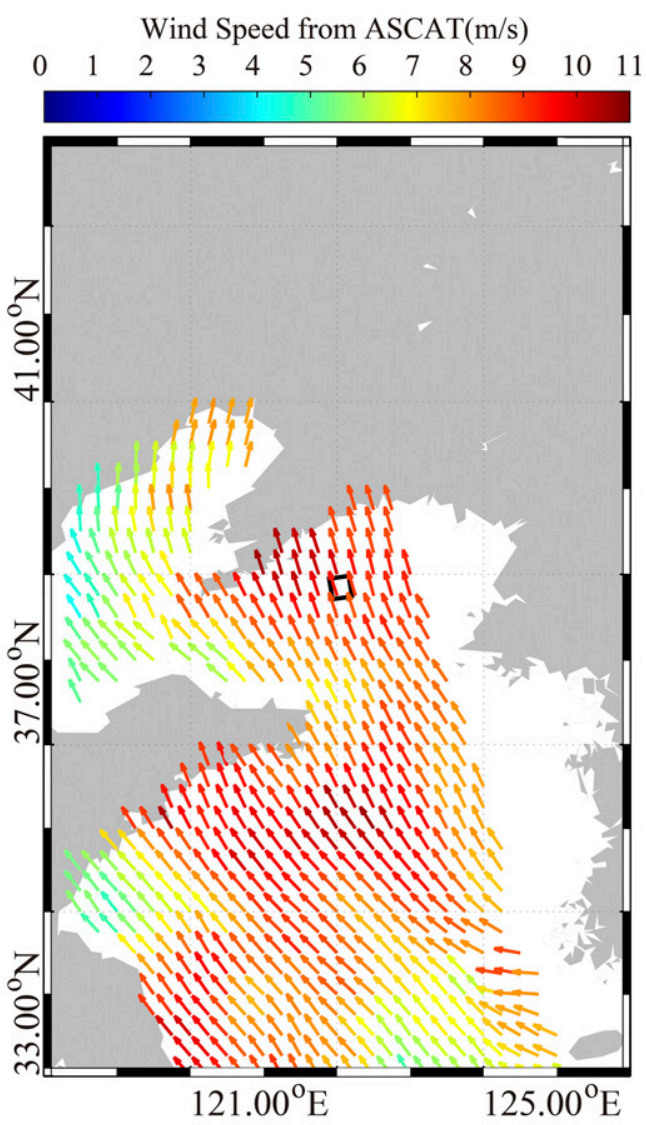

(b)

FIG. 3. (a) A GF-3 SAR calibrated image collected at 0955 UTC 3 Aug 2017, around the Bohai Sea; (b) the corresponding ASCAT wind map in descending orbit acquired at 0930 UTC, in which the black-outlined rectangle represents the spatial coverage of the GF-3 SAR image.

GMFs are presented in section 4. Section 5 discusses results, and conclusions are summarized in section 6.

\section{Description of datasets}

In the past two years, the $G F-3$ mission collected more than 10000 images in WV and QPS modes. Some 1023 images in QPS mode were collected from the China seas and others in WV mode were collected over global seas. Each level-1 (L-1) image product was converted into an NRCS image according to this calibration formula:

$$
\sigma^{0}=(\mathrm{DN})^{2}\left(\frac{M}{32767}\right)^{2}-N
$$

where $\sigma^{0}$ represents the NRCS in decibels, DN is the intensity of a pixel, and $M$ and $N$ are the constants stored in the annotated file with the original SAR intensity image. Note that the updated constants $M$ and $N$ were officially released by National Ocean Satellite Application
Center (NSOAS) and were derived from a large number of images collected over the Amazon rain forest.

ECMWF is a valuable source for atmospheric and marine research, which has provided continuous global atmospheric-marine hindcast products since 1979, including wind vector at $10 \mathrm{~m}$ above sea surface and wave parameters. The ECMWF winds are released at 6-h intervals each day (i.e., 0000, 0600, 1200 and 1800 UTC), which are widely used for tuning (Hersbach et al. 2007; Shao et al. 2016) and validating (Hersbach 2010; Stoffelen et al. 2017) wind retrieval algorithms from SAR images. As an example, the GF-3 SAR image with homogenous wind streaks acquired in QPS mode at 0926 UTC 31 March 2017 is shown in Fig. 1, in which colored arrows denote the $0.125^{\circ}$ gridded ECMWF reanalysis wind vectors at 0600 UTC overlaid on the VV-polarization image.

The GF-3 SAR imagery has a fine spatial resolution, including an 8-m standard pixel for QPS mode and a 10-m pixel for $\mathrm{WV}$ mode, and the spatial resolution of the ECMWF reanalysis winds is about $12.5 \mathrm{~km}$. 


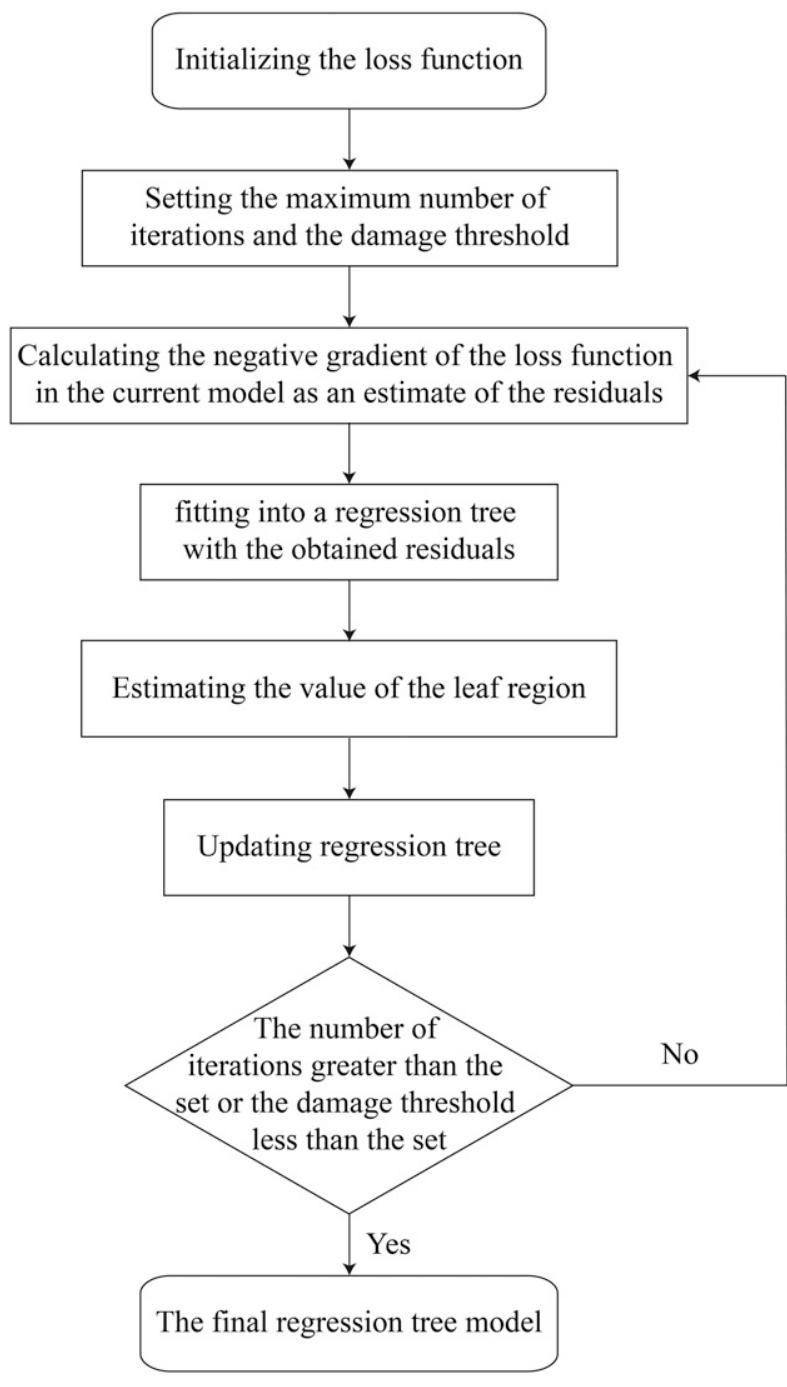

FIG. 4. Flowchart of the GBDT model.

Therefore, we divided each image into a number of subscenes with a spatial coverage of about $4 \mathrm{~km}^{2}$. In addition, we interpolated the ECMWF winds over the temporal scale to $1 \mathrm{~h}$ using a cubic spline (Wang et al. 2018). Hence, the time difference between GF-3 SAR imaging and the ECMWF winds was within $30 \mathrm{~min}$. The available dataset included ECMWF wind vectors, the SAR-measured NRCS at four channels, and incidence angle and azimuth angle of the subscenes covering the ECMWF grids, which we used to develop the wind retrieval algorithm. The histogram of the wind speed and incidence angle and rose diagram of the wind direction form ECMWF are shown in Fig. 2, in which the wind speed was up to $25 \mathrm{~m} \mathrm{~s}^{-1}$. There are limited samples at wind speeds greater than $16 \mathrm{~m} \mathrm{~s}^{-1}$, however, and the incidence angle of the dataset ranged primarily from $30^{\circ}$ to $42^{\circ}$.

The measurements from the Advanced Scatterometer (ASCAT) on board $\mathrm{Met} O p-A / B$ are reliable open access data that can be used to validate the SAR-derived winds. The comparison between ASCAT winds and in situ buoy measurements has shown a root-mean-square error (RMSE) of $1.8 \mathrm{~m} \mathrm{~s}^{-1}$ for wind speed (Bello-Orgaz et al. 2016). Figure 3a presents a VV-polarization $G F-3$ case that shows an image collected at 0955 UTC 3 August 2017, in the Bohai Sea. The corresponding ASCAT wind map in the descending orbit was taken at 0930 UTC (see Fig. 3b; the black-outlined rectangle represents the spatial coverage of the GF-3 SAR image). We also observed that homogenous wind streaks were visible, which was generally consistent with ASCAT wind directions, similar to the previous case.

\section{Method}

In this section, the wind retrieval problem is addressed through the regression estimation. The latter can be performed using many artificial intelligence methods, (e.g., linear regression, logistic regression, polynomial regression, Bayesian, $K$ nearest neighbors, and random forest). In this study, we selected the last three methods as intelligent wind retrieval algorithms.

\section{a. Gradient boosting decision tree}

Gradient boosting decision tree (GBDT) is a popular boosting model. GBDT trains the newly added weak classifier according to the negative gradient information of the loss function of the current model and then combines the trained weak classifier into the existing model in the form of accumulation. GBDT is an iterative learning model based on the residual of a decision tree, which embodies the idea of learning from mistakes.

As a nonlinear regression algorithm, GBDT is also suitable for sea surface wind speed retrieval. The application of GBDT to wind speed retrieval includes three steps. 1) Initialize

$$
f_{0}(x)=\arg \min \sum_{i=1}^{N} L\left(y_{i}, c\right),
$$

where $f_{0}()$ is a tree with only roots, $N$ is the number of samples, $L()$ is the loss function, $y_{i}$ is the output of the $i$ th sample, and $c$ is the output of the current regression tree. 2) For $m=1,2, \ldots, M$ :

(i) For $i=1,2, \ldots, N$ compute

$$
\gamma_{i m}=-\left\{\frac{\partial L\left[y_{i}, f\left(x_{i}\right)\right]}{\partial f\left(x_{i}\right)}\right\}_{f=f_{m-1}},
$$

where $f\left(x_{i}\right)$ is the $i$ th regression tree and $\gamma_{i m}$ is the gradient of the loss function against the current model. 


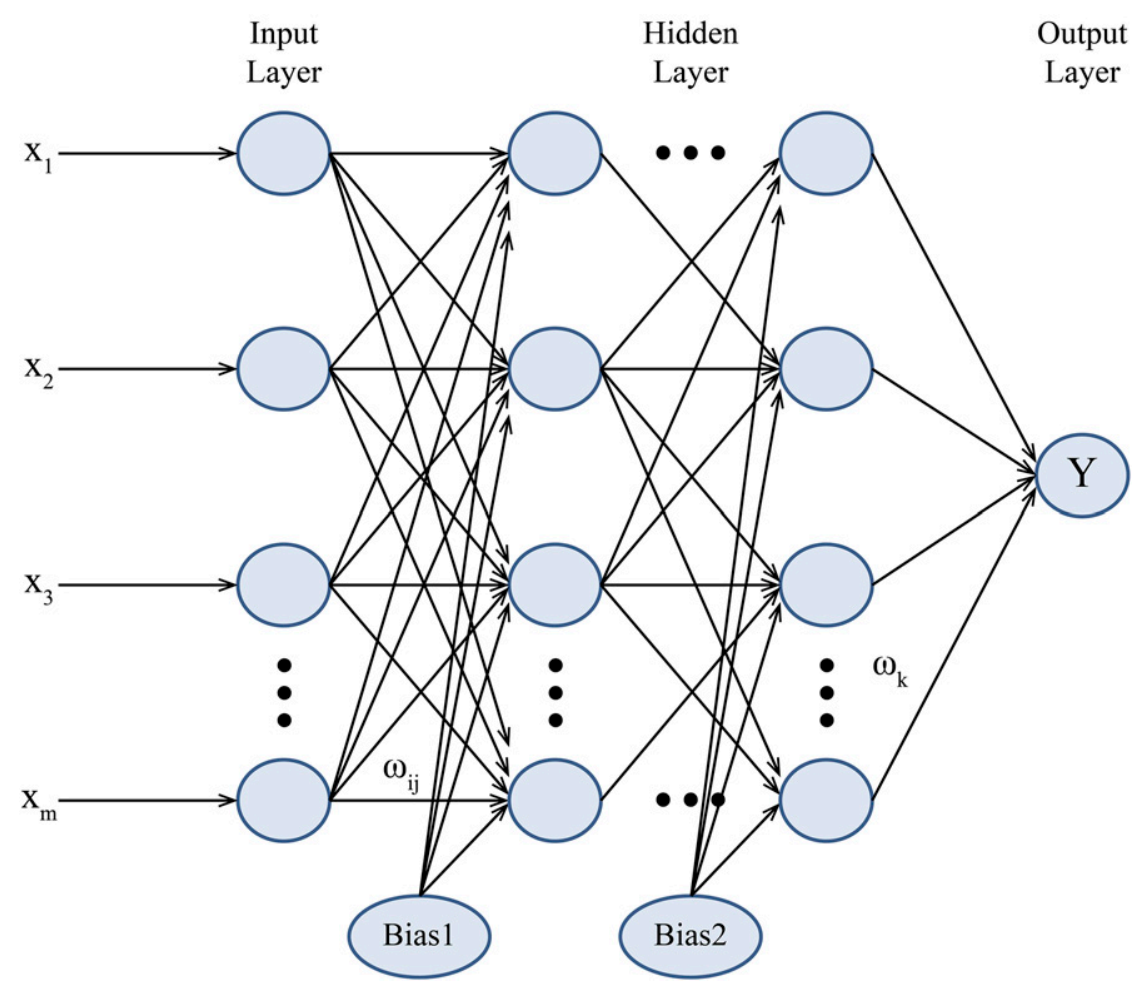

FIG. 5. Flowchart of the network structure.

(ii) Fit a regression tree to the targets $\gamma_{i m}$ giving terminal regions, as follows,

$$
\gamma_{j m}, \quad j=1,2, \ldots, J_{m}
$$

(iii) For $j=1,2, \ldots, J_{m}$ compute

$$
c_{j m}=\arg \min _{c} \sum_{x_{j} \in R_{j m}} L\left[y_{i}, f_{m-1}\left(x_{i}\right)+c\right]
$$

where $c_{j m}$ is the output value of the $m$ th regression tree in the leaf node region $R_{j m}$.

(iv) Update the function stated as

$$
f_{m}(x)=f_{m-1}(x)+\sum_{j=1}^{J_{m}} c_{j m} I\left(x \in R_{j m}\right) .
$$

The last step 3) yields the output $\hat{f}(x)$, given by

$$
\hat{f}(x)=f_{M}(x) .
$$

Figure 4 shows the flowchart of the GBDT algorithm.

\section{b. Back-propagation neural network}

A back-propagation neural network (BPNN) is trained according to the error back propagation algorithm and is a supervised learning algorithm. It has been proved mathematically that BPNN has the ability to realize any complex nonlinear mapping, which makes it particularly suitable for solving the complex mechanism of wind speed retrieval. The network can automatically extract reasonable solving rules through supervised learning prior knowledge; that is, it has a self-learning ability. The network has the ability to generalize the different data of a new scene compared with the other wind speed retrieval methods based on models. The basic network structure is shown in Fig. 5.

The BPNN used in this study consisted of an input layer, a hidden layer and an output layer. The input layer of the network had seven nodes, and the input variables were the four polarization channels, wind direction from ECMWF, incident angle, and azimuth angle. Considering that the range of input data varied significantly, the input data had to be normalized so that the value range of each feature was in the interval $[0,1]$. The hidden layer had three layers, each layer had 64 nodes, and the activation function used a rectified linear unit (ReLU). The output layer had one node, and the output was the retrieved wind speed.

The learning process of the network consists of seven steps: 1) Initialize the weight and bias of the network. 2) Randomly select a group of samples for forward propagation of the network, and calculate the output of each 


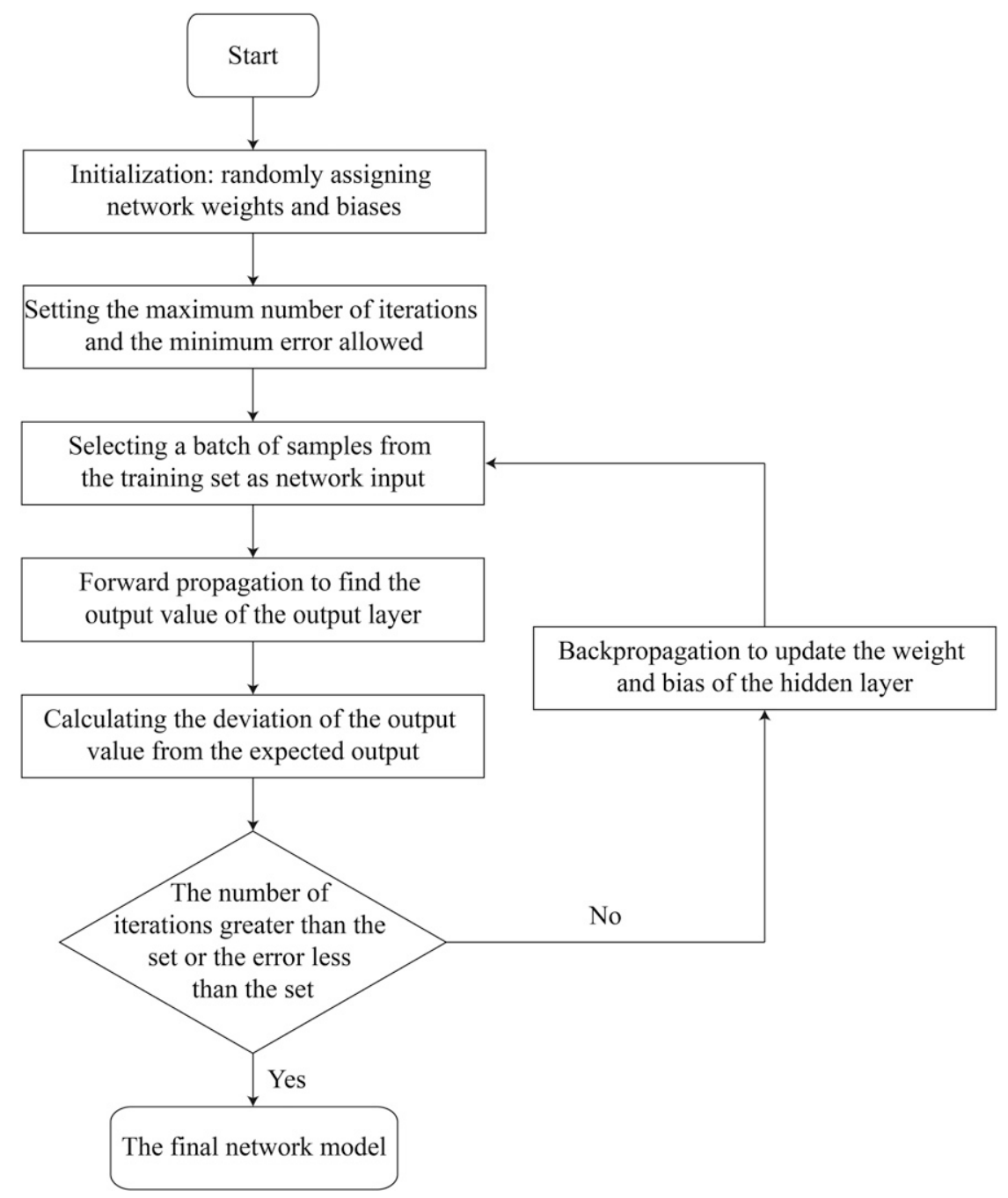

FIG. 6. Flowchart of the PNN model.

neuron in the hidden layer successively. The network used sigmoid as the activation function, and its function form is shown in the following equation:

$$
f(x)=\frac{1}{1+e^{-x}} .
$$

The output of each neuron in the hidden layer is as follows:

$$
\begin{aligned}
a_{j} & =\sum_{i=1}^{n} w_{i j} x_{i}+b_{i} \\
Z_{j} & =f\left(a_{j}\right),
\end{aligned}
$$

where $a_{j}$ represents the input variable of the $j$ th node of the $Z$ th hidden layer of the neural network, $n$ represents the number of nodes of the input layer, $w_{i j}$ represents the connection weight of the $i$ th node of the input layer and the $j$ th node of the hidden layer, $b_{i}$ represents the connection bias of the $i$ th node of the input layer and the $j$ th node of the hidden layer, and $Z_{j}$ represents the output variable of the $j$ th node of the hidden layer. 3) Calculate the output of the output layer neurons:

$$
Y=\sum_{k=1}^{m} w_{k} o_{k}+b_{k}
$$

where $Y$ represents the output variable of the network, $w_{k}$ represents the weight between the neurons connected to the output, $o_{k}$ represents the output value of the $k$ th neuron of the hidden layer $O$, and $b_{k}$ represents the bias between the neurons connected to the output. 4) Calculate the mean square error $L$ of the network output value $Y$ and the true value $T$ : 


$$
L=\frac{1}{n_{i=1}} \sum_{i}^{n}\left(Y_{i}\right)^{2}
$$

where $L$ represents the mean square error of the output value and the true value, and $n$ represents the total number of nodes. 5) If $L$ is less than the training target error or reaches the maximum number of training iterations, the training is stopped; otherwise, continue to step 6. 6) Calculate the error gradient and update the weights and bias as follows:

$$
\begin{aligned}
\Delta w_{i j} & =-\eta \frac{\partial L(w, b)}{\partial w_{i j}}, \\
\Delta b_{j} & =-\eta \frac{\partial L(w, b)}{\partial b_{j}}, \\
w_{i j} & =w_{i j}-\Delta w_{i j}, \quad \text { and }, \\
b_{j} & =b_{j}-\Delta b_{j},
\end{aligned}
$$

where $\eta$ represents the learning rate. Similarly, the weights and bias of each layer of the network can be updated. 7) Return to step 2 until the training error $L$ is less than the maximum number of trainings reached, at which point stop the training. Figure 6 shows the flowchart of the BPNN algorithm.

\section{c. K nearest neighbors}

The $K$ nearest neighbors (KNN) is an algorithm that stores all available cases and predicts the numerical target based on a similarity measure, such as Euclidean distance, Manhattan distance, or Hamming distance. In general, the voting method can be used in the regression estimation or classification task; that is, the category tag that appears most in $k$ samples can be selected as the prediction result. In the regression task, the average method can be used; that is, the average value of the output of $k$ samples can be used as the prediction result. The core steps that illustrate wind retrieval using KNN are as follows: 1) calculate the distance between the new point and each training point, 2) select the closest $k$ data points on the basis of the Euclidean distances, and 3) use the average of these data points as the final prediction for the new point. Figure 7 shows the flowchart of the KNN algorithm.

\section{d. Random forest}

The random forest (RF) model is used to build a forest in a random way. There are many decision trees in the forest, and the decision trees are not related to each other. When there is a new sample, we let each decision

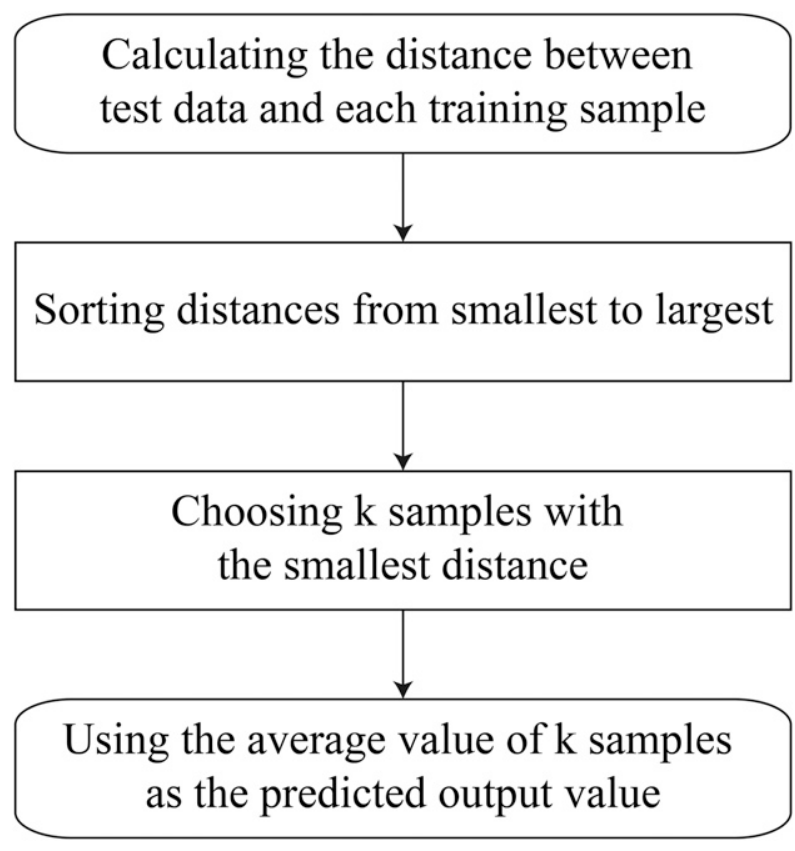

FIG. 7. Flowchart of the KNN model.

tree in the forest make a separate judgment to see which category this sample belonged to. Then we used the method of voting as the final classification result. In the regression problem, we used RF to take the average of the output values of all decision trees as the final prediction result.

The commonly used regression tree algorithm is the least squares regression tree generation algorithm. The input of the algorithm is the training dataset $S$, and the output is the regression tree $f(x)$. In the input space where the training dataset is located, the algorithm recursively divides each region into two subregions and determines the output value on each subregion to construct a binary decision tree.

The application of RF algorithm in wind speed retrieve includes four steps. 1) Use bootstrap to sample the original training set $S$ to generate a training set $S_{i}$. 2) Use $S_{i}$ to generate an unpruned tree $T_{i}$ :

(i) Select the optimal segmentation variable $j$ and the segmentation point $s$ to solve

$$
\min _{j, s}\left[\min _{c_{1}} \sum_{x_{i} \in R_{1}(j, s)}\left(y_{i}-c_{1}\right)^{2}+\min _{c_{2}} \sum_{x_{i} \in R_{2}(j, s)}\left(y_{i}-c_{2}\right)^{2}\right] \text {, }
$$

in which $j$ is the cut variable, $s$ is the cut node, and $y_{i}$ is the output of $x_{i}$, as follows,

$$
c_{1}=\frac{1}{N_{1}} \sum_{i \in N_{1}(j, s)} y_{i}
$$




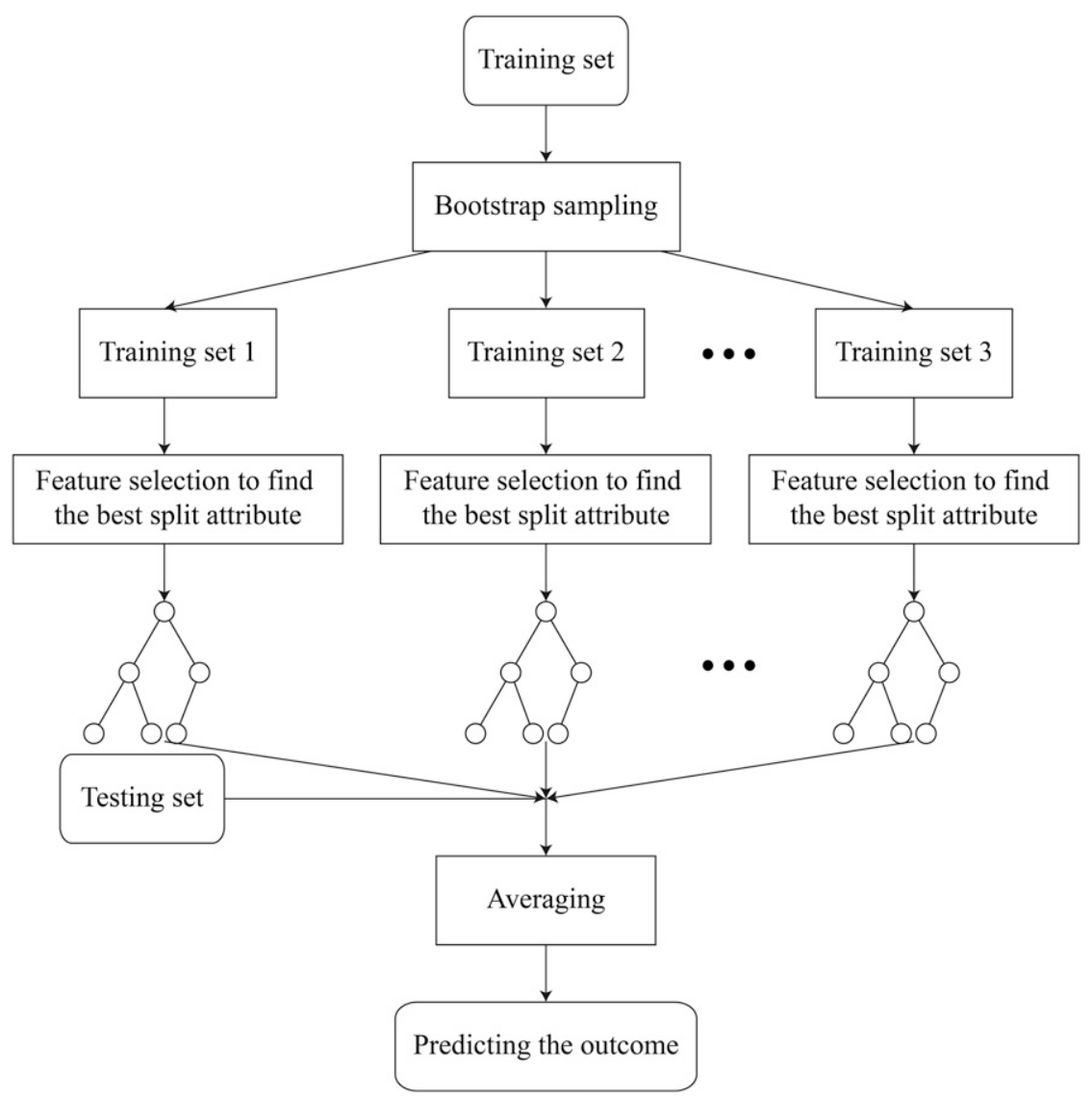

FIG. 8. Flowchart of the RF model.

$$
c_{2}=\frac{1}{N_{2 x_{i}} \in N_{2}(j, s)} \sum_{i},
$$

where $N_{1}$ and $N_{2}$ represent the number of samples in region $R_{1}$ and $R_{2}$, respectively.

(ii) Use the selected pair $(j, s)$ to divide the region and determine the corresponding output value, as follows,

$$
\begin{aligned}
R_{1}(j, s) & =\left\{x \mid x^{(j)} \leq s\right\}, \quad R_{2}(j, s)=\left\{x \mid x^{(j)}>s\right\}, \\
\hat{c}_{m} & =\frac{1}{N_{m} x_{i} \in R_{m}(j, s)} y_{i}, \quad x \in R_{m}, \quad m=1,2 .
\end{aligned}
$$

(iii) Repeat steps $\mathrm{i}$ and ii for the two subareas until the stop condition is met.

(iv) Divide the input space into $M$ areas: $R_{1}, R_{2}, \ldots$, $R_{M}$, and generate the decision tree, as follows,

$$
f(x)=\sum_{m=1}^{M} \hat{c}_{m} I\left(x \in R_{m}\right) \text {. }
$$

The next step consists of 3 ) repeating step 2 to generate the set of trees $\left\{h_{i}, i=1,2, \ldots, n\right\}$. 4) For test sample $x_{t}$, the decision tree $h_{i}$ output is $h_{i}\left(x_{t}\right)$. The final prediction result is $f\left(x_{t}\right)$, which is calculated according to the following formula:

$$
f\left(x_{t}\right)=\frac{1}{N} \sum_{i=1}^{N} h_{i}\left(x_{t}\right)
$$

Figure 8 shows the flowchart of the RF algorithm.

\section{e. Development of intelligent wind retrieval algorithm}

We randomly divided the dataset into 10 subsets. We used nine of these subsets (37845 samples) as training sets to train the neural networks and used one (4205 samples) as the validation set to test the effectiveness of the network. Figure 9 shows the polarized NRCSs at four channels of matchups versus wind speeds from ECMWF. The color represents the sample numbers. We observed a linear relationship between the NRCS at cross-polarization channels and wind speed at a $10-\mathrm{m}$ height above the sea surface $U_{10}$, which indicated that the noise equivalent sigma zero of $G F-3$ SAR data acquired in cross polarization was approximately $-40 \mathrm{~dB}$. This kind of behavior was consistent with findings from two studies proposed by Ren et al. (2017) and 


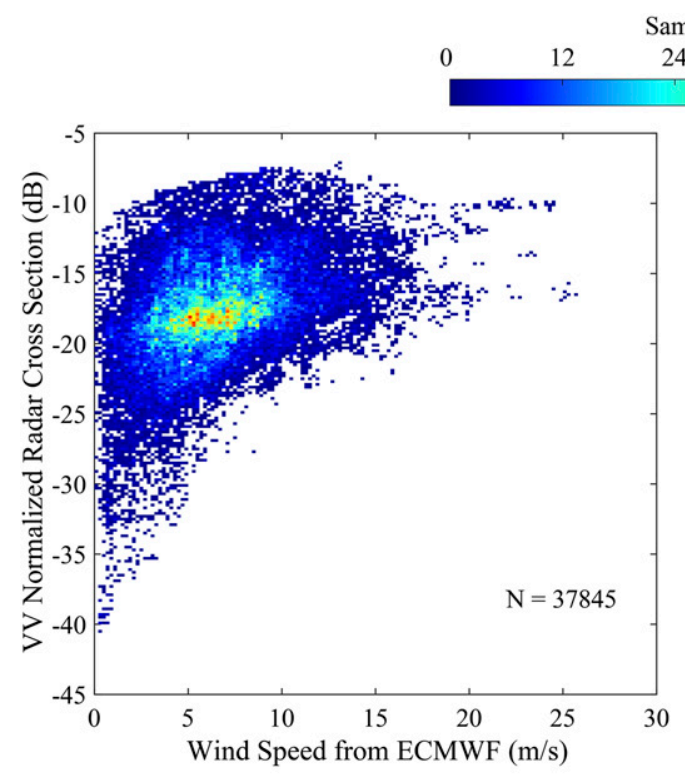

(a)

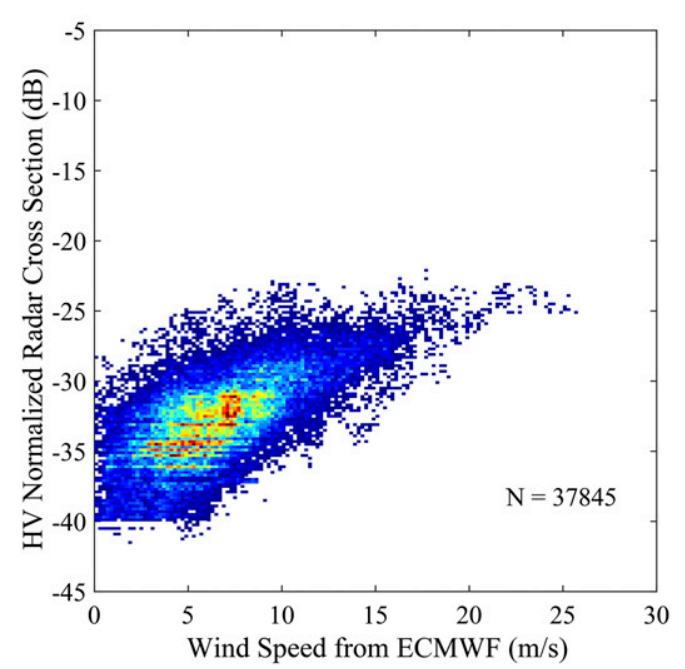

(c)

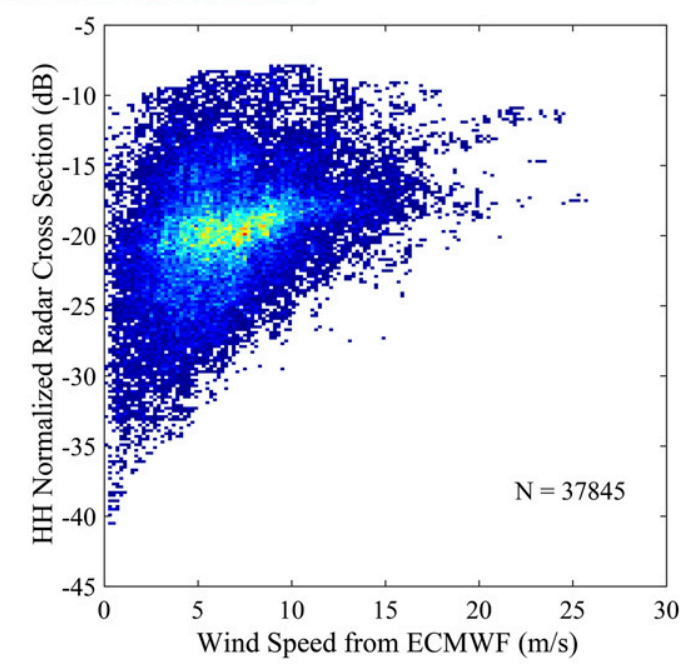

(b)

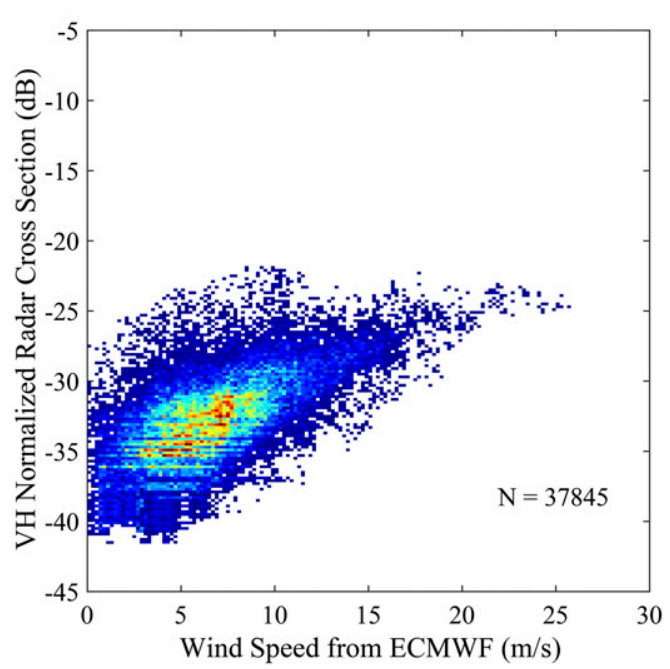

(d)

FIG. 9. The relation between SAR-measured NRCSs at four channels and ECMWF wind speeds: (a) VV-polarization, (b) HH-polarization, (c) HV-polarization, and (d) VH-polarization.

Zhang et al. (2019). Therefore, we thought that crosspolarization NRCS was useful for wind retrieval from the $G F-3$ SAR image.

According to the development of GMF, a wind vector can be determined by two variables through an empirical model, including VV-polarization NRCS and incidence angle. Different research groups have exploited a set of GMFs, although they have had the same basic function (Masuko et al. 1986). These GMFs have demonstrated different performance for various SAR sensors. We believed that the accuracy of retrieval could be improved when including more relevant information in a retrieval algorithm.
It also has been shown that the relationship between copolarization NRCS and wind speed (like an exponential function of wind speed) is different from that between cross-polarization NRCS and wind speed (like a linear function of wind speed); therefore, it was difficult to include NRCS at four channels in an empirical function. Under this circumstance, we tried to develop an intelligent wind retrieval algorithm based on artificial neural network using the NRCSs at four channels, all of which were directly related to the wind.

Again, the training data inputs included the four polarization NRCSs, wind direction from ECMWF, incidence 


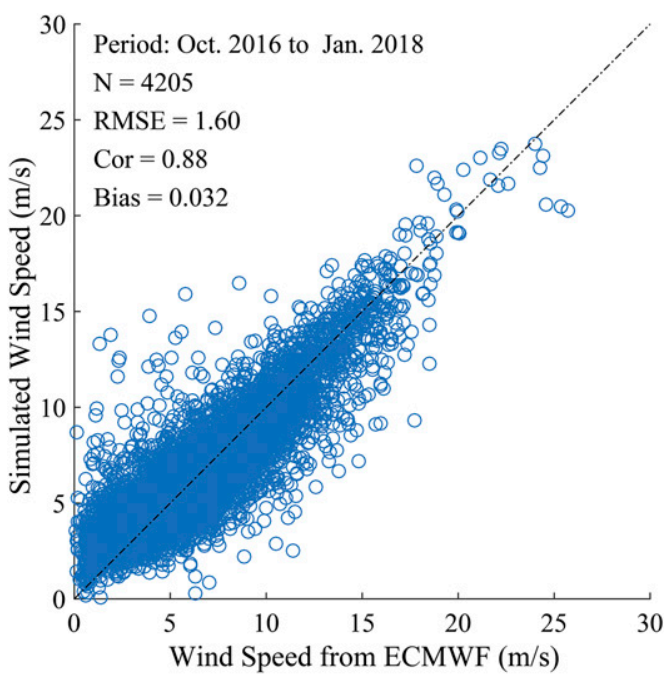

(a)

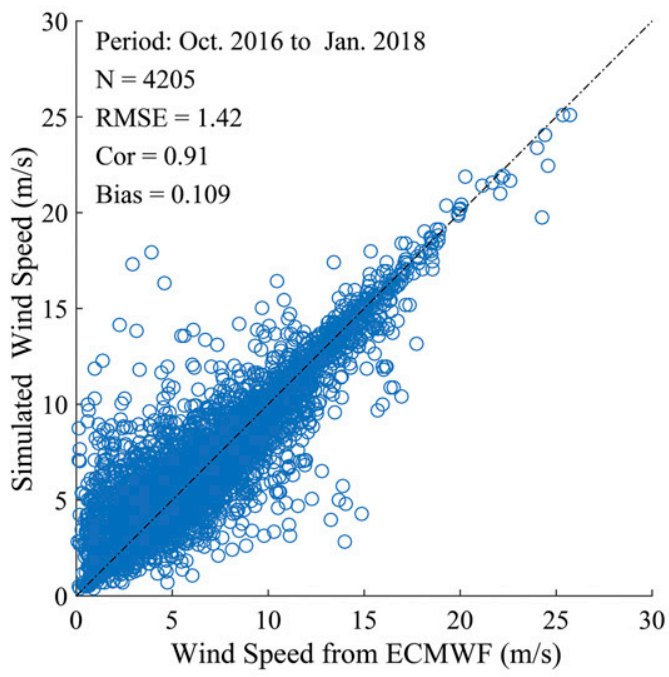

(c)

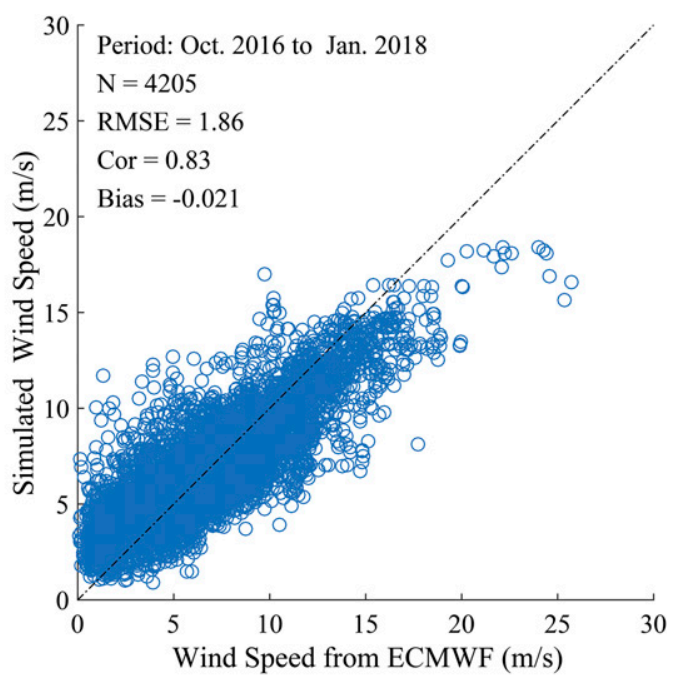

(b)

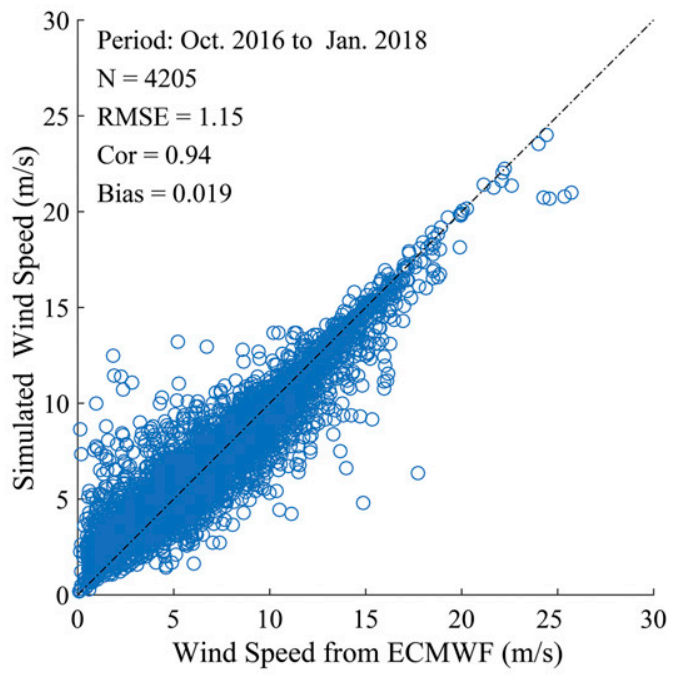

(d)

FIG. 10. ECMWF winds vs retrievals obtained using (a) GBDT, (b) BPNN, (c) KNN, and (d) RF.

angle, azimuth angle and wind speed from ECMWF. We evaluated the performance of the models by comparing retrieved sea surface wind speed values with ECMWF data. Figure 10 shows the retrievals obtained using the four models (i.e., GBDT, BPNN, KNN, and RF) in comparison with wind speed from ECMWF. We found that RF resulted in the best performance [i.e., a 0.94 correlation (COR) and a $\left.1.15 \mathrm{~m} \mathrm{~s}^{-1} \mathrm{RMSE}\right]$. Thus, we selected the RF model for wind speed retrieval using quad-polarization GF-3 SAR images, which we denote as QPWIND_GF.

\section{Results}

We validated the wind speeds retrieved using QPWIND_GF against wind speeds from ASCAT.
In the retrieval process, we set SAR-measured NRCS at four channels, wind direction from ECMWF, incidence angle and azimuth angle as the inputs, and the output was the retrieved wind speed. As an example, the retrieval wind map for the example in Fig. 1 is shown in Fig. 11. In this case, the retrieved wind speed using QPWIND_GF covering the ASCAT wind grid $\left(=10 \mathrm{~m} \mathrm{~s}^{-1}\right)$ was $11.0 \mathrm{~m} \mathrm{~s}^{-1}$. Figure 12 shows the statistical analysis of the proposed QPWIND_GF with ASCAT measurements. Results showed a $0.74 \mathrm{~m} \mathrm{~s}^{-1}$ RMSE of wind speed, which was less than a $1.94 \mathrm{~m} \mathrm{~s}^{-1}$ standard deviation of wind speed using C-SARMOD for VV-polarization GF-3 SAR validated against ASCAT winds, as presented in our recent study (Shao et al. 2019). Under this circumstance, we improved 


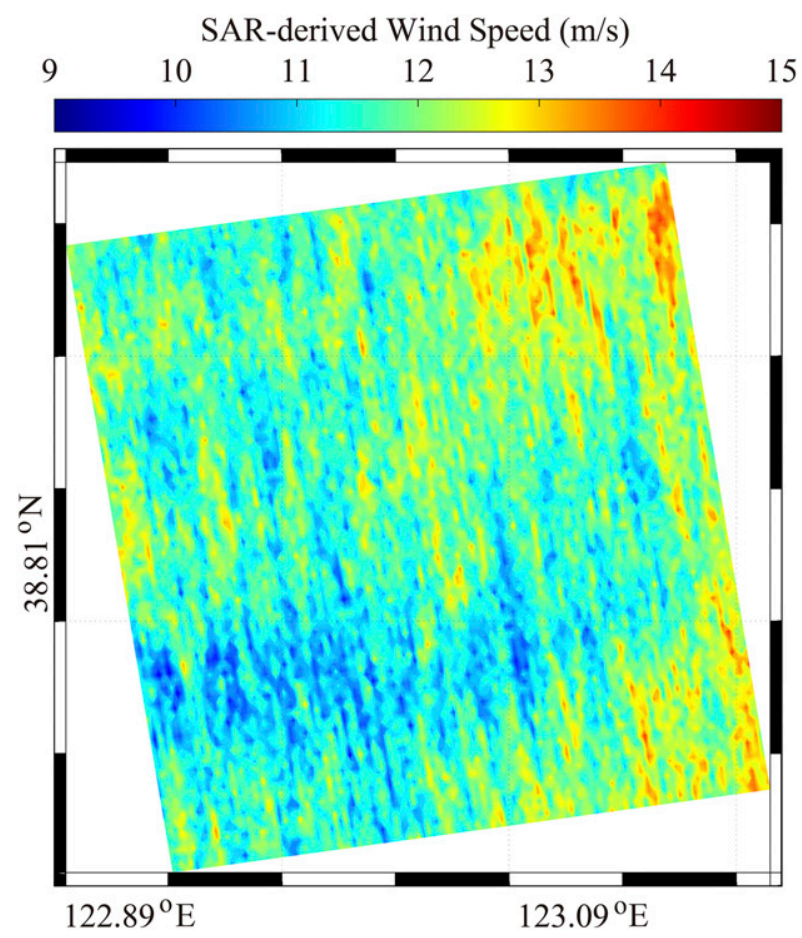

FIG. 11. The retrieved wind map for the GF-3 SAR image collected at 0955 UTC 3 Aug 2017, around the Bohai Sea using QPWIND_GF.

the accuracy of SAR-derived wind speed using QPWIND_GF.

We also compared ASCAT measurements using the newly released wind retrieval algorithms for copolarization GF-3 SAR, including CMOD7 and C-SARMOD2. The formulation of CMOD7 was a lookup table, which we derived from a large amount of NRCS measured from scatterometer and auxiliary winds from numeric model and observations (Stoffelen et al. 2017). C-SARMOD2 was an empirical function and the model was tuned from SAR-measured NRCS and collocated winds from moored buoys ( $\mathrm{Lu}$ et al. 2018). The retrieval wind maps using CMOD7 and C-SARMOD2 for the example in Fig. 1 are exhibited in Figs. 13 and 14, respectively. Figure 15 shows a $1.88 \mathrm{~m} \mathrm{~s}^{-1}$ RMSE of wind speed using CMOD7 and a $1.98 \mathrm{~m} \mathrm{~s}^{-1}$ RMSE of wind speed using C-SARMOD2. It is not surprising that both CMOD7 and C-SARMOD2 were less accurate than QPWIND_GF; in fact, QPWIND_GF included the SAR-measured NRCS at four channels.

\section{Discussion}

We demonstrated the applicability of the proposed QPWIND_GF under various conditions. The bias

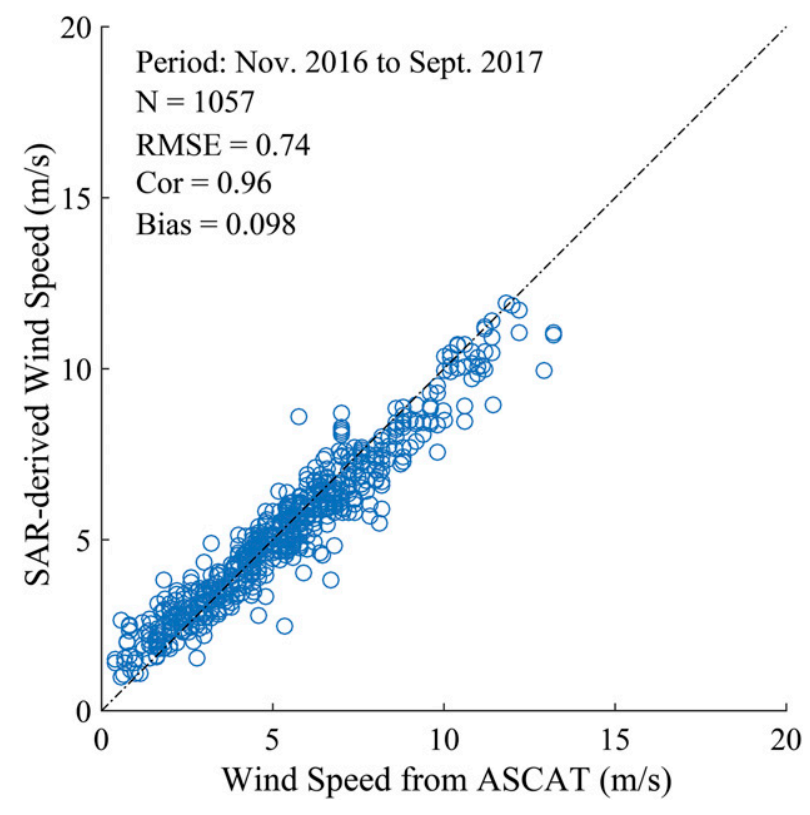

FIG. 12. SAR-derived wind speeds using QPWIND_GF vs observed wind speeds from ASCAT.

(i.e., SAR-derived wind speed minus observed wind speed from ASCAT) versus the incidence angle and the wind direction (i.e., the true wind direction from ASCAT minus SAR azimuth angle) are shown in Figs. 16-18

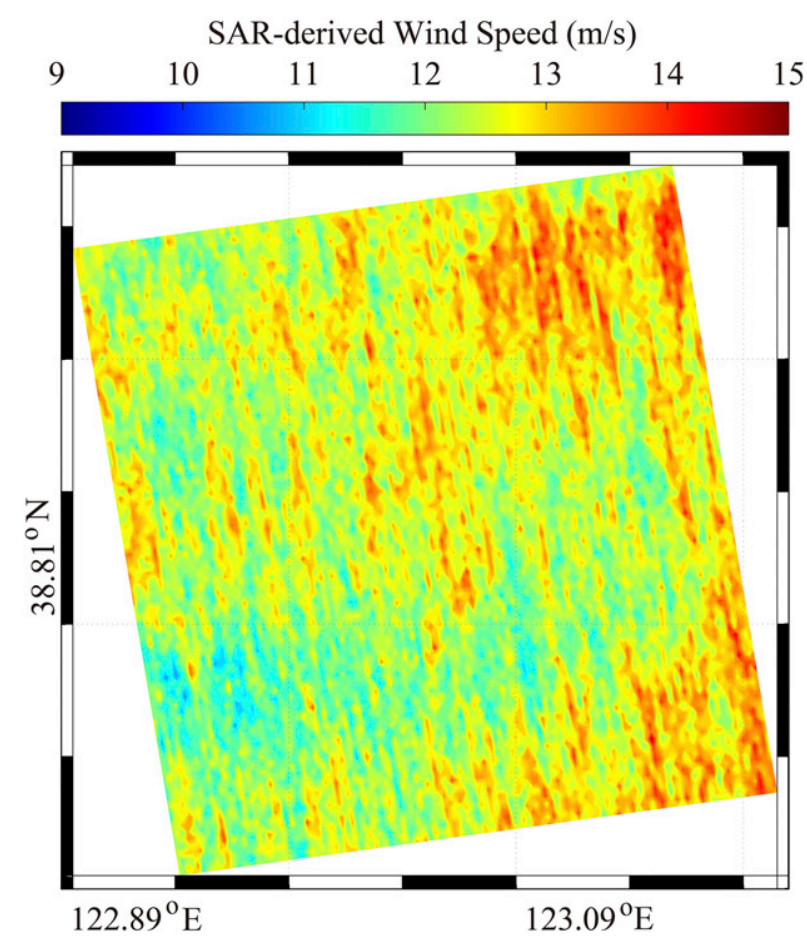

FIG. 13. The wind map retrieved by processing the $G F-3 \mathrm{SAR}$ image collected at 0927 UTC 1 Jan 2017, around the Yellow Sea using CMOD7. 


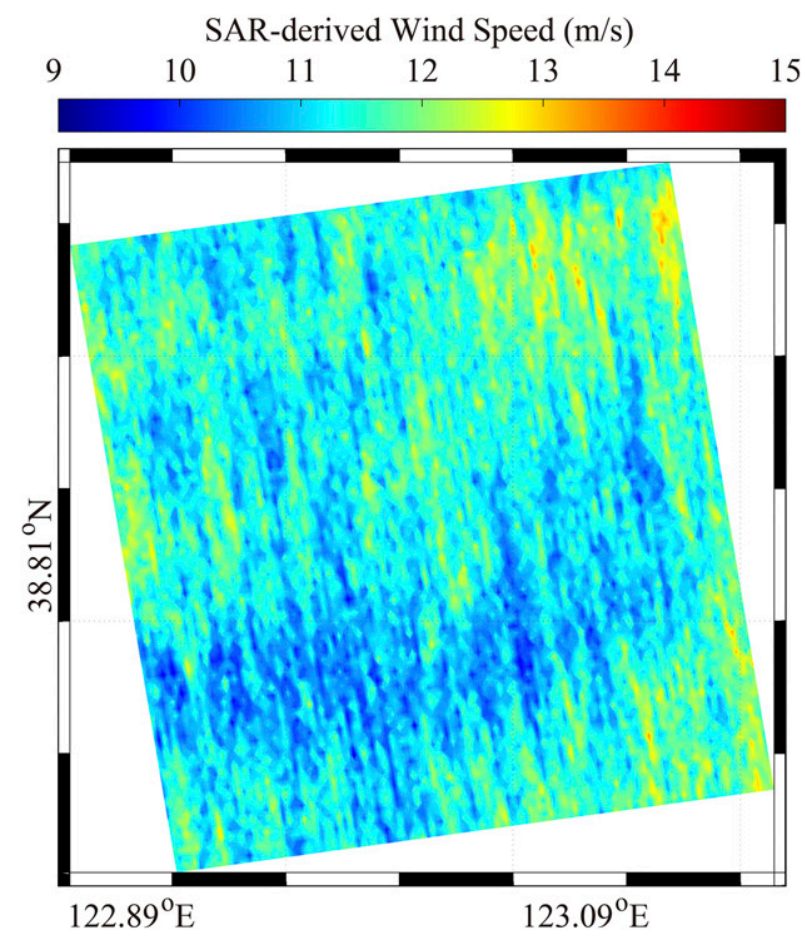

FIG. 14. As in Fig. 13, but using C-SARMOD2.

[in panels (a) and (b) of each figure], which respectively correspond to the results using QPWIND_GF, CMOD7, and C-SARMOD2. We used a bin size of $2^{\circ}$ for the incidence angle and $40^{\circ}$ for wind direction to group data pairs. The error bars represent the standard deviation of each bin. In general, the variation of bias using QPWIND_GF improved significantly.

The variation of bias as a function of wind speed along with the ASCAT wind for a bin size of $1 \mathrm{~m} \mathrm{~s}^{-1}$ is presented in Figs. 16c, 17c, and 18c. We observed that the retrieved wind speed had a large deviation at low wind (wind speed less than $2 \mathrm{~ms}^{-1}$ ); however, the bias $\left(=1.5 \mathrm{~m} \mathrm{~s}^{-1}\right)$ using QPWIND_GF was less than that speed $\left(=3 \mathrm{~m} \mathrm{~s}^{-1}\right)$ using the other two GMFs under such conditions. Bragg waves at the sea surface quantitatively decreased under low wind conditions, causing a relatively smooth sea surface roughness. In other words, the SAR backscattering signal was weak at low wind speeds. This is the probable explanation for the decreasing accuracy with decreasing wind speed less than $2 \mathrm{~m} \mathrm{~s}^{-1}$. Nevertheless, it was evident that the retrieval error remained at a specific value with increasing wind speed (e.g., $1.5 \mathrm{~m} \mathrm{~s}^{-1}$ for CMOD7 and $2 \mathrm{~m} \mathrm{~s}^{-1}$ for C-SARMOD2). We resolved this issue in the improved QPWIND_GF algorithm, which indicated $\pm 0.5 \mathrm{~m} \mathrm{~s}^{-1}$ of the retrieved wind speed. ECMWF data generally underestimate wind data (Stopa and Cheung 2014), which we used to tune the proposed algorithm, causing the observed underestimation of retrieved results.

\section{Conclusions}

At present, a wind retrieval algorithm has been developed for SAR at a single-polarization channel (e.g., copolarization and cross polarization). It is well known,

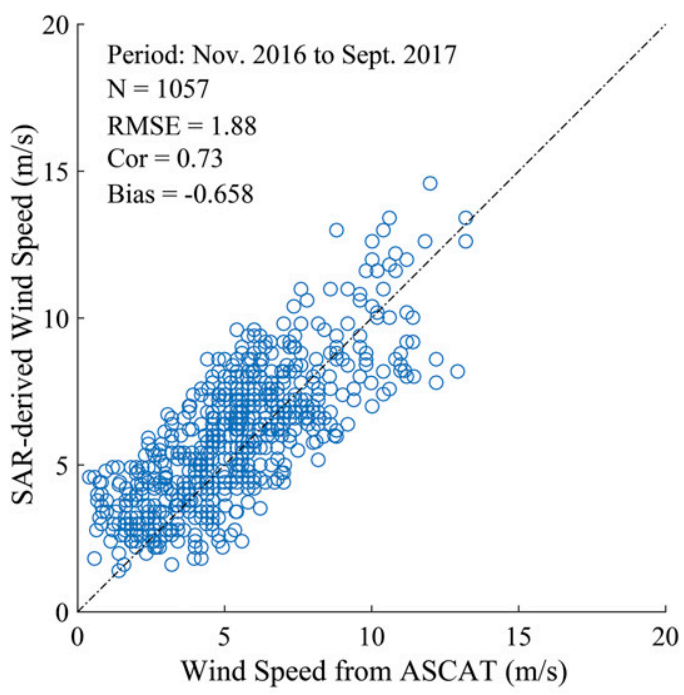

(a)

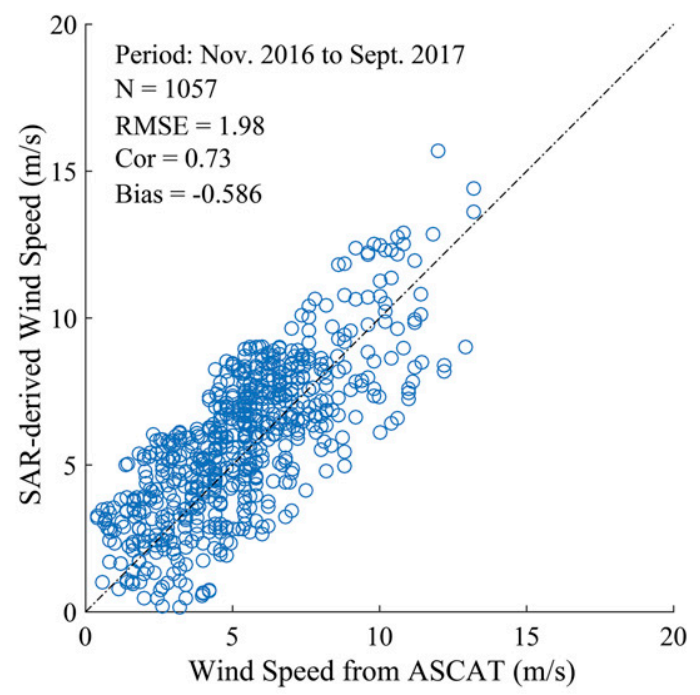

(b)

FIG. 15. (a) Comparison of SAR-derived wind speeds using (a) CMOD7 or (b) C-SARMOD2 vs observed wind speeds from ASCAT. 


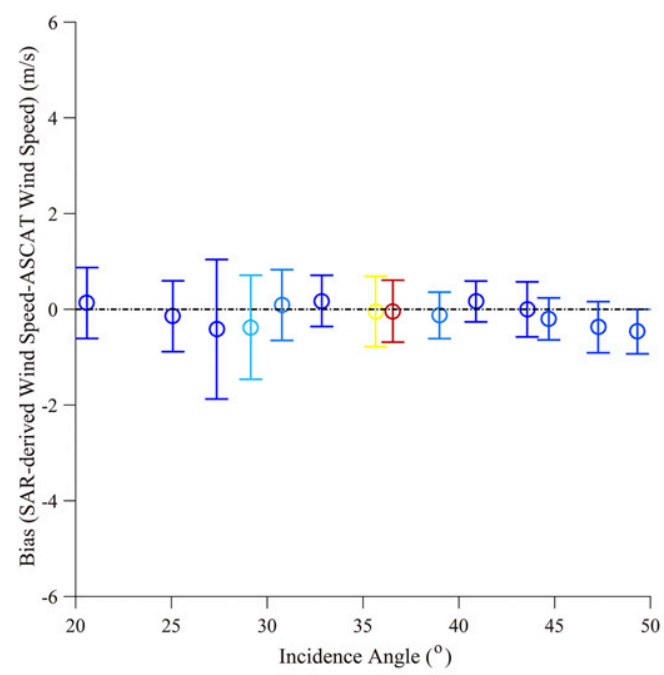

(a)

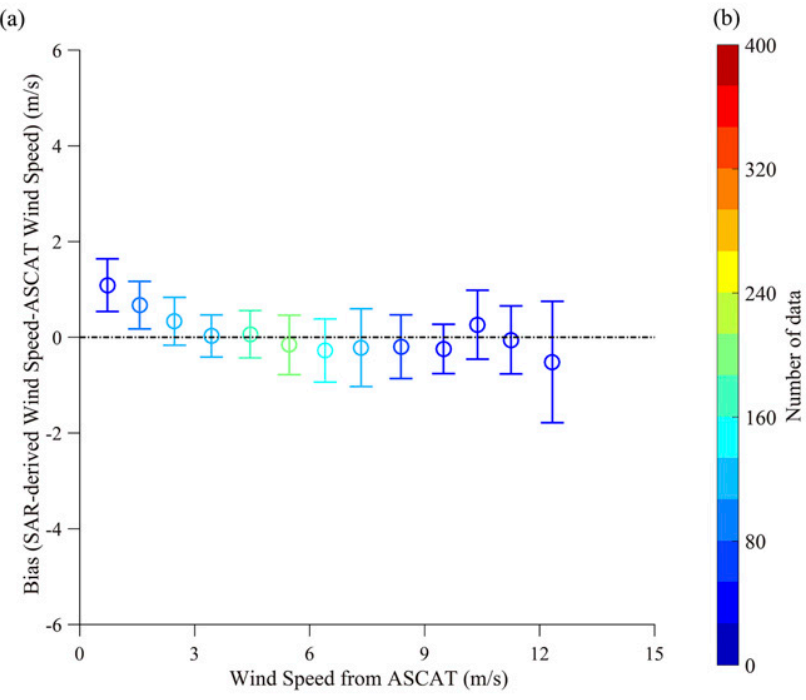

(c)

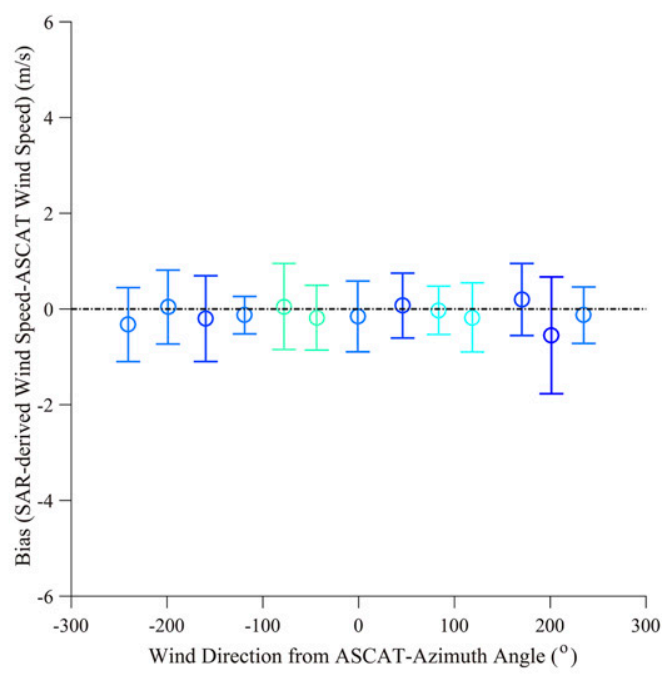

(b)

FIG. 16. Bias between SAR-derived wind speeds using QPWIND_GF and observed wind speeds from ASCAT vs three parameters: (a) SAR incidence angles, (b) difference between ASCAT wind directions and SAR azimuth angles, and (c) ASCAT wind speeds.

however, that the wind vector is related to both co- and cross-polarization NRCS (Mouche and Chapron 2015). Therefore, we believed that the accuracy of wind speed could be improved using an SAR backscattering signal at four channels. In this study, we confirmed the applicability of an artificial neural network for wind retrieval from $G F-3$ SAR images. We selected four existing artificial models with a neural network method: GBDT, BPNN, KNN, and RF.

We used more than 4000 matchups, of which $90 \%$ (about 37845 samples) were used to tune the four models, and the rest were used to test the efficiency of these models. The results achieved a 0.94 COR by the RF model (renamed QPWIND_GF), which was better than that of other models. We acquired several images in QPS mode, which were collocated with ASCAT wind, and processed using QPWIND_GF. The retrieved wind speeds validated against the measurements from ASCAT showed a $0.74 \mathrm{~m} \mathrm{~s}^{-1}$ RMSE. Moreover, we tested the two advanced wind retrieval algorithms for copolarization SAR (i.e., CMOD7 and C-SARMOD2) for $G F-3$ SAR, and both showed around $1.9 \mathrm{~m} \mathrm{~s}^{-1}$ RMSE of wind speed, which had a similar accuracy for Sentinel-1A SAR (Lu et al. 2018). The analysis also confirmed that the SAR-derived wind speed had a stable trend $\left( \pm 0.5 \mathrm{~m} \mathrm{~s}^{-1}\right)$ at wind speeds greater than $2 \mathrm{~m} \mathrm{~s}^{-1}$ when using the QPWIND_GF algorithm. Under these circumstance, we proved that the artificial neural 


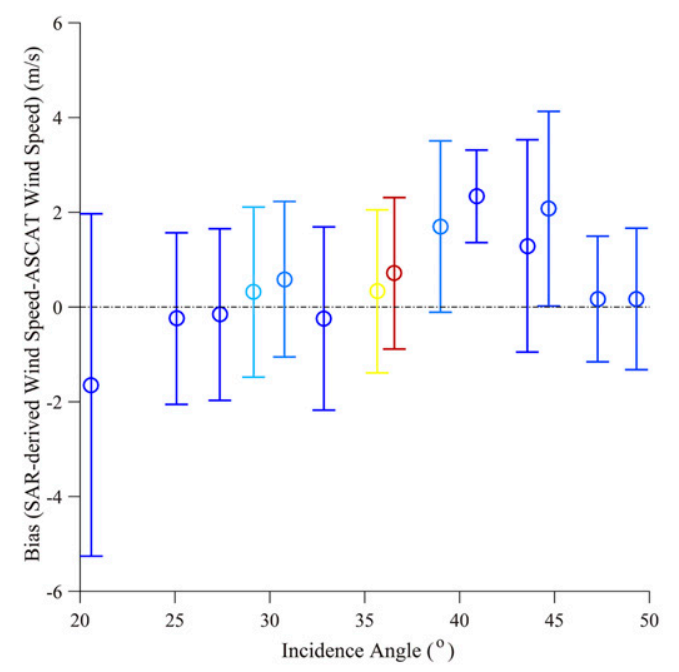

(a)

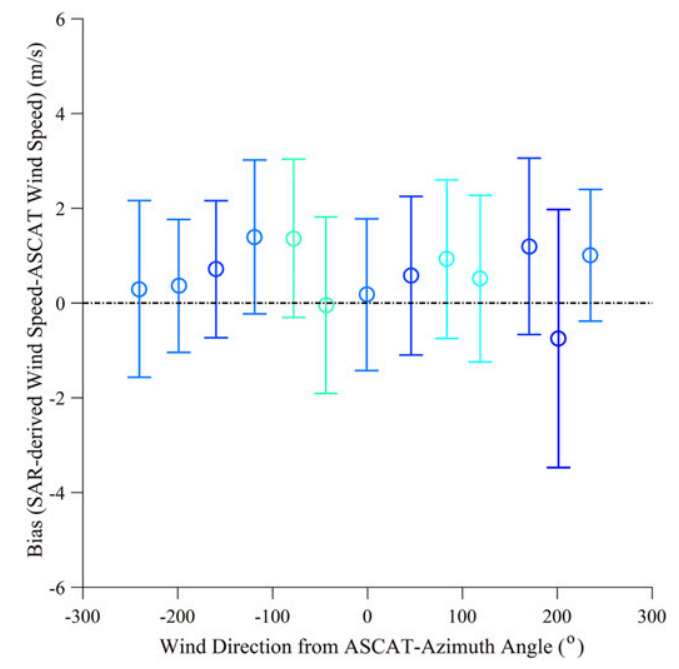

(b)

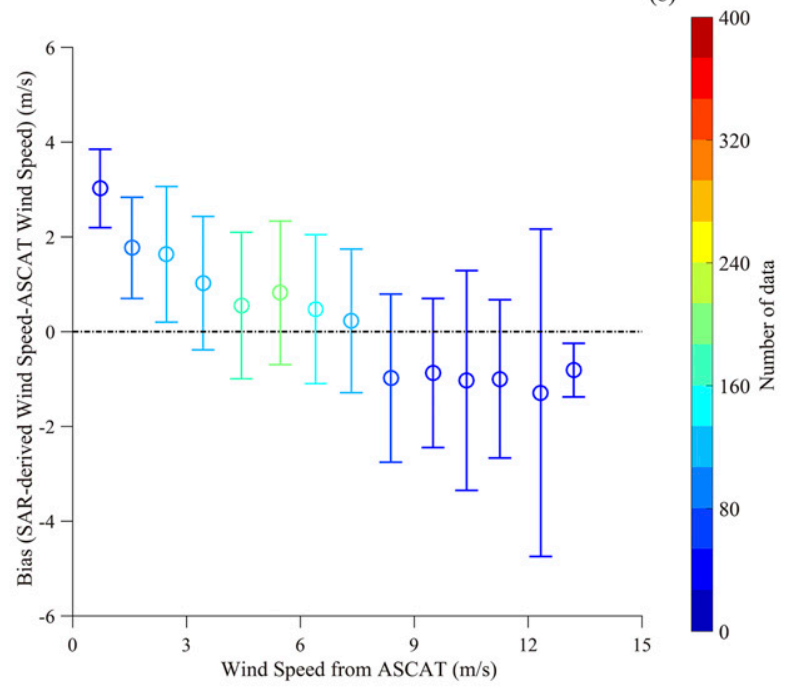

(c)

FIG. 17. As in Fig. 16, but for the bias between SAR-derived wind speeds using CMOD7 and observed wind speeds from ASCAT.

network method had the advanced feasibility for the SAR marine application. We also anticipated that a better result would be achieved using more $G F-3$ SAR images, because the QPWIND_GF algorithm relied on the background dataset.

Monitoring of strong winds (probably greater than $25 \mathrm{~m} \mathrm{~s}^{-1}$ ) is an interesting topic for the remote sensing community. In the near future, we will adopt the neutral network method for wind retrieval from $G F-3$ SAR images in typhoons and hurricanes.

Acknowledgments. The authors acknowledge support from the National Key Research and Development Program of China under Grant 2017YFA0604901, the National Natural Science Foundation of China un- der Grants 41806005 and 41776183, the Public Welfare Technical Applied Research Project of Zhejiang Province of China under Contract LGF19D060003, and the Science and Technology Project of Zhoushan City under Contract 2019C21008. The C-band Gaofen-3 synthetic aperture radar images can be accessed online (at http://dds.nsoas.org.cn) through an authorized account issued by the National Ocean Satellite Application Center (NSOAS), and investigators abroad can access the GF-3 data through international cooperation, including the ESA-MOST Dragon (http:// dragon4.esa.int/page_home.php) and CFOSAT programs. The wind products from the Advanced Scatterometer at $0.25^{\circ}$ grids are available online (http://archive.eumetsat.int) through an authorized 


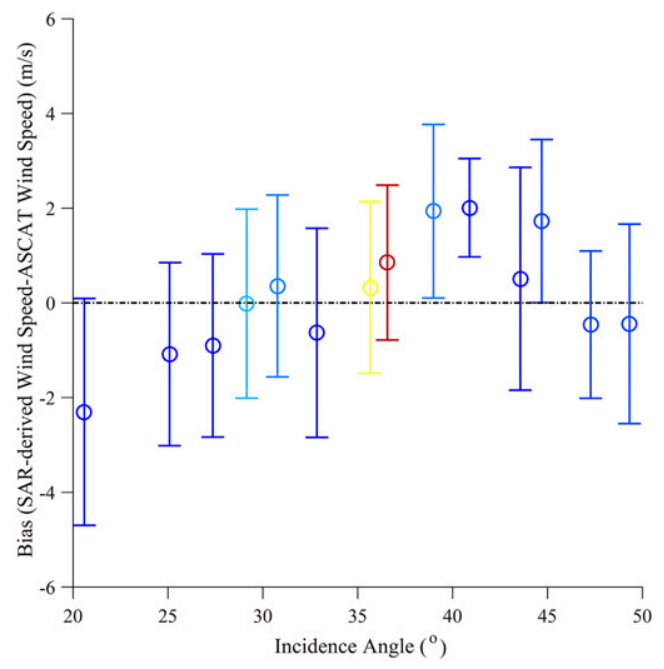

(a)

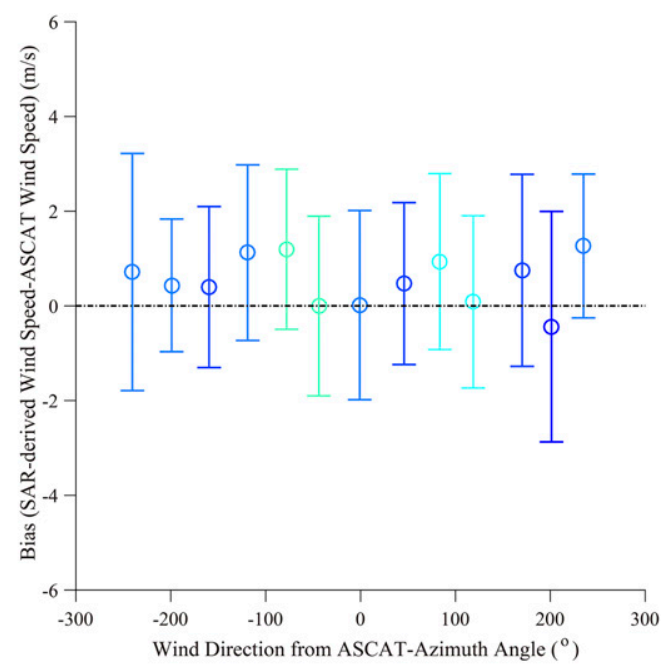

(b)

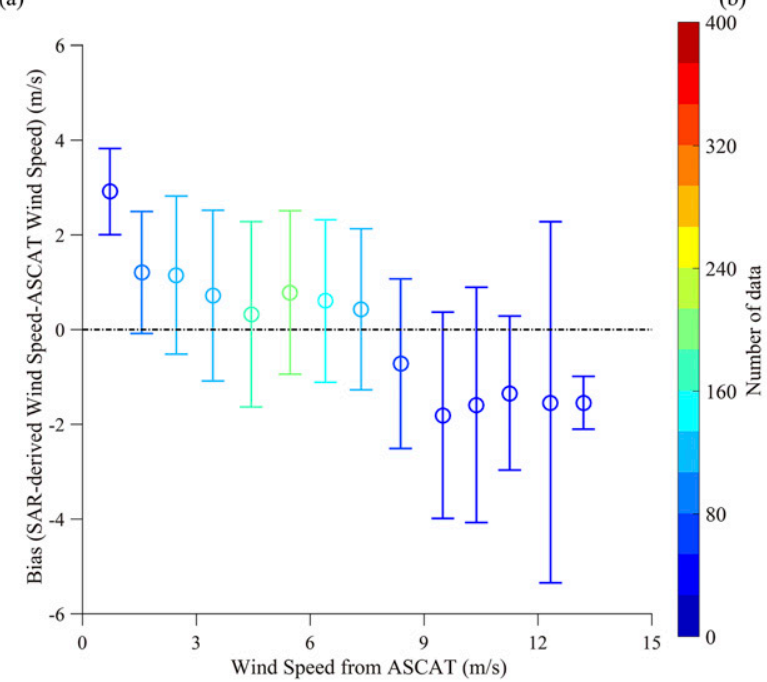

(c)

FIG. 18. As in Fig. 16, but for the bias between SAR-derived wind speeds using C-SARMOD2 and observed wind speeds from ASCAT.

account. The European Centre for Medium-Range Weather Forecasts reanalysis wind data at a $0.125^{\circ}$ grid are openly available online (http://www.ecmwf.int).

\section{REFERENCES}

Alpers, W., and B. Brümmer, 1994: Atmospheric boundary layer rolls observed by the synthetic aperture radar aboard the ERS-1 satellite. J. Geophys. Res., 99, 12 613-12 621, https:// doi.org/10.1029/94JC00421.

Bello-Orgaz, G., J. J. Jung, and D. Camacho, 2016: Social big data: Recent achievements and new challenges. Inf. Fusion, 28, 45-59, https://doi.org/10.1016/j.inffus.2015.08.005.

Benassai, G., D. Luccio, V. Corcione, F. Nunziata, and M. Migliaccio, 2018: Marine spatial planning using high resolution SAR measurements. IEEE J. Oceanic Eng., 43, 586-594, https:// doi.org/10.1109/JOE.2017.2782560.
Chapron, B., H. Johnsen, and R. Garello, 2001: Wave and wind retrieval from SAR images of the ocean. Ann. Telecommun., 56, 682-699, https://doi.org/10.1007/BF02995562.

Corcione, V., F. Nunziata, and M. Migliaccio, 2018: Megi typhoon monitoring by $\mathrm{X}$-band synthetic aperture radar measurements. IEEE J. Oceanic Eng., 43, 184-194, https://doi.org/ 10.1109/JOE.2017.2700558.

_ , G. Grieco, M. Portabella, F. Nunziata, and M. Migliaccio, 2019: A novel azimuth cut-off implementation to retrieve sea surface wind speed from SAR imagery. IEEE Trans. Geosci. Remote Sens., 57, 3331-3340, https://doi.org/10.1109/ TGRS.2018.2883364.

Fois, F., P. Hoogeboom, F. L. Chevalier, and A. Stoffelen, 2015: Future ocean scatterometry: On the use of crosspolar scattering to observe very high winds. IEEE Trans. Geosci. Remote Sens., 53, 5009-5020, https://doi.org/10.1109/ TGRS.2015.2416203.

Grieco, G., W. Lin, M. Migliaccio, F. Nirchio, and M. Portabella, 2016: Dependency of the Sentinel-1 azimuth wavelength 
cut-off on significant wave height and wind speed. Int. J. Remote Sens., 37, 5086-5104, https://doi.org/10.1080/ 01431161.2016 .1226525 .

Hersbach, H., 2010: Comparison of C-Band scatterometer CMOD5.N equivalent neutral winds with ECMWF. J. Atmos. Oceanic Technol., 27, 721-736, https://doi.org/10.1175/ 2009JTECHO698.1.

__- A. Stoffelen, and S. D. Haan, 2007: An improved C-band scatterometer ocean geophysical model function: CMOD5. J. Geophys. Res., 112, C03006, https://doi.org/10.1029/ 2006JC003743.

Horstmann, J., W. Koch, S. Lehner, and R. Tonboe, 2000: Wind retrieval over the ocean using synthetic aperture radar with C-band HH polarization. IEEE Trans. Geosci. Remote Sens., 38, 2122-2131, https://doi.org/10.1109/36.868871.

Huang, L. Q., B. Liu, X. F. Li, Z. H. Zhang, and W. X. Yu, 2017 Technical evaluation of Sentinel-1 IW mode cross-pol radar backscattering from the ocean surface in moderate wind condition. Remote Sens., 9, 854, https://doi.org/10.3390/ rs9080854.

Hwang, P. A., B. Zhang, J. V. Toporkov, and W. Perrie, 2010: Comparison of composite Bragg theory and quad-polarization radar backscatter from Radarsat-2: With applications to wave breaking and high wind retrieval. J. Geophys. Res., 115 C08019, https://doi.org/10.1029/2009JC005995.

- , A. Stoffelen, G. J. Van Zadelhoff, W. Perrie, B. Zhang, H. Li, and H. Shen, 2015: Cross-polarization geophysical model function for C-band radar backscattering from the ocean surface and wind speed retrieval. J. Geophys. Res. Oceans, 120, 893-909, https://doi.org/10.1002/2014JC010439.

Lin, H., Q. Xu, and Q. A. Zheng, 2008: An overview on SAR measurements of sea surface wind. Prog. Nat. Sci., 18, 913-919, https://doi.org/10.1016/J.PNSC.2008.03.008.

Lu, Y., B. Zhang, W. Perrie, A. A. Mouche, X. F. Li, and H. Wang, 2018: A C-band geophysical model function for determining coastal wind speed using synthetic aperture radar. IEEE J. Sel. Top. Appl. Earth Obs. Remote Sens., 11, 2417-2428, https:// doi.org/10.1109/JSTARS.2018.2836661.

Masuko, H., K. Okamoto, M. Shimada, and S. Niwa, 1986: Measurement of microwave backscattering signatures of the ocean surface using $\mathrm{X}$ band and Ka band airborne scatterometers. J. Geophys. Res., 91, 13 065-13083, https://doi.org/10.1029/ JC091IC11P13065.

Monaldo, F., C. Jackson, X. F. Li, and W. G. Pichel, 2016: Preliminary evaluation of Sentinel-1A wind speed retrievals. IEEE J. Sel. Top. Appl. Earth Obs. Remote Sens., 9, 2638-2642, https:// doi.org/10.1109/JSTARS.2015.2504324.

Mouche, A. A., and B. Chapron, 2015: Global C-Band Envisat, Radarsat-2 and Sentinel-1 SAR measurements in copolarization and cross-polarization. J. Geophys. Res. Oceans, 120, 7195-7207, https://doi.org/10.1002/2015JC011149.

_, D. Hauser, J. F. Daloze, and C. Guerin, 2005: Dual-polarization measurements at C-band over the ocean: Results from airborne radar observations and comparison with Envisat ASAR data. IEEE Trans. Geosci. Remote Sens., 43, 753-769, https://doi.org/ 10.1109/TGRS.2005.843951.

Quilfen, Y., A. Bentamy, T. Elfouhaily, K. Katsaros, and J. Tournadre, 1998: Observation of tropical cyclones by highresolution scatterometry. J. Geophys. Res., 103, 7767-7786, https://doi.org/10.1029/97JC01911.

Ren, L., J. S. Yang, A. A. Mouche, H. Wang, J. Wang, G. Zheng, and H. Zhang, 2017: Preliminary analysis of Chinese GF-3 SAR quad-polarization measurements to extract winds in each polarization. Remote Sens., 9, 1215, https://doi.org/10.3390/ rs9121215.

Shao, W. Z., J. Sun, C. L. Guan, and Z. F. Sun, 2014: A method for sea surface wind field retrieval from SAR image mode data. J. Ocean Univ. China, 13, 198-204, https://doi.org/10.1007/s11802-014-1999-5.

—, Z. Zhang, X. M. Li, and W. L. Wang, 2016: Sea surface wind speed retrieval from TerraSAR-X HH-polarization data using an improved polarization ratio model. IEEE J. Sel. Top. Appl. Earth Obs. Remote Sens., 9, 4991-4997, https://doi.org/ 10.1109/JSTARS.2016.2590475.

, Y. X. Sheng, and J. Sun, 2017: Preliminary assessment of wind and wave retrieval from Chinese Gaofen-3 SAR imagery. Sensors, 17, 1705, https://doi.org/10.3390/s17081705.

—, X. Z. Yuan, Y. Sheng, J. Sun, W. Zhou, and Q. J. Zhang, 2018: Development of wind speed retrieval from cross-polarization Chinese Gaofen-3 synthetic aperture radar in typhoons. Sensors, 18, 412, https://doi.org/10.3390/s18020412.

-, S. Zhu, J. Sun, X. Z. Yuan, Y. X. Sheng, Q. J. Zhang, and Q. Y. Ji, 2019: Evaluation of wind retrieval from copolarization Gaofen-3 SAR imagery around China seas. J. Ocean Univ. China, 18, 80-92, https://doi.org/10.1007/ S11802-019-3779-8.

Stoffelen, A., and D. Anderson, 1997: Scatterometer data interpretation: Derivation of the transfer function, CMOD4. J. Geophys. Res., 102, 5767-5780, https://doi.org/10.1029/ 96JC02860.

—_ J. A. Verspeek, J. Vogelzang, and A. Verhoef, 2017: The CMOD7 geophysical model function for ASCAT and ERS wind retrievals. IEEE J. Sel. Top. Appl. Earth Obs. Remote Sens., 10, 2123-2134, https://doi.org/10.1109/ JSTARS.2017.2681806

Stopa, J. E., and K. F. Cheung, 2014: Intercomparison of wind and wave data from the ECMWF reanalysis interim and the NCEP Climate Forecast System Reanalysis. Ocean Modell., 75, 65-83, https://doi.org/10.1016/j.ocemod.2013.12.006.

Vachon, P. W., and F. W. Dobson, 2000: Wind retrieval from Radarsat SAR images: Selection of a suitable C-band $\mathrm{HH}$ polarization wind retrieval model. Can. J. Remote Sens., 26, 306-313, https://doi.org/10.1080/07038992.2000.10874781.

, and J. Wolfe, 2011: C-band cross-polarization wind speed retrieval. IEEE Geosci. Remote Sens. Lett., 8, 456-459, https:// doi.org/10.1109/LGRS.2010.2085417.

Voronovich, A. G., and V. U. Zavorotny, 2014: Full-polarization modeling of monostatic and bistatic radar scattering from a rough sea surface. IEEE Trans. Antennas Propag., 62, 1362-1371, https://doi.org/10.1109/TAP.2013.2295235.

Wang, L., B. Han, X. Z. Yuan, B. Lei, C. B. Ding, Y. L. Yao, and Q. Chen, 2018: A preliminary analysis of wind retrieval, based on GF-3 wave mode data. Sensors, 18, 1604, https://doi.org/ $10.3390 / \mathrm{s} 18051604$

Yang, X. F., X. F. Li, W. G. Pichel, and Z. W. Li, 2011: Comparison of ocean surface winds from Envisat ASAR, MetOp ASCAT scatterometer, buoy measurements, and NOGAPS model. IEEE Trans. Geosci. Remote Sens., 49, 4743-4750, https:// doi.org/10.1109/TGRS.2011.2159802.

Zhang, B., and W. Perrie, 2012: Cross-polarized synthetic aperture radar: A new potential measurement technique for hurricanes. Bull. Amer. Meteor. Soc., 93, 531-541, https://doi.org/10.1175/ BAMS-D-11-00001.1.

, - — , and Y. J. He, 2011: Wind speed retrieval from Radarsat-2 quad-polarization images using a new polarization ratio model. J. Geophys. Res., 116, C08008, https://doi.org/10.1029/ 2010JC006522. 
Zhang, G. S., X. F. Li, W. Perrie, P. A. Hwang, and X. F. Yang, 2017: A hurricane wind speed retrieval model for C-band Radarsat-2 cross-polarization ScanSAR images. IEEE Trans. Geosci. Remote Sens., 55, 4766-4774, https://doi.org/10.1109/ TGRS.2017.2699622.

Zhang, T. Y., X. M. Li, Q. Feng, Y. Z. Ren, and Y. S. Shi, 2019: Retrieval of sea surface wind speeds from Gaofen-3 full polarimetric data. Remote Sens., 11, 813, https://doi.org/10.3390/ rs11070813.
Zhou, L. Z., G. Zheng, X. F. Li, J. S. Yang, L. Ren, P. Chen, H. G. Zhang, and X. L. Lou, 2017: An improved local gradient method for sea surface wind direction retrieval from SAR imagery. Remote Sens., 9, 671, https://doi.org/ 10.3390/rs9070671.

Zhu, S., and Coauthors, 2018: Evaluation of Chinese quadpolarization Gaofen-3 SAR wave mode data for significant wave height retrieval. Can. J. Remote Sens., 44, 588-600, https://doi.org/10.1080/07038992.2019.1573136. 